\title{
Weighted inequalities for quasilinear integral operators on the semiaxis and application to the Lorentz spaces
}

\author{
Dmitrii V. Prokhorov, Vladimir D. Stepanov*
}

\begin{abstract}
Weighted $L^{p}-L^{r}$ inequalities with arbitrary measurable non-negative weights for positive quasilinear integral operators with Oinarov's kernel on the semiaxis are characterized. Application to the boundedness of maximal operator in the Lorentz $\Gamma$-spaces is given.
\end{abstract}

2000 Mathematics Subject Classification: Primary 26D15; Secondary 47G10

Key words and phrases: Weighted inequality, quasilinear operator, Lebesgue space, Lorentz space.

\section{Introduction}

Let $\mathbb{R}_{+}:=[0, \infty)$. Denote $\mathfrak{M}$ the set of all measurable functions on $\mathbb{R}_{+}$and $\mathfrak{M}^{+} \subset \mathfrak{M}$ the subset of all non-negative functions. If $0<p \leq \infty$ and $v \in \mathfrak{M}^{+}$we define

$$
\begin{gathered}
L_{v}^{p}:=\left\{f \in \mathfrak{M}:\|f\|_{L_{v}^{p}}:=\left(\int_{0}^{\infty}|f(x)|^{p} v(x) d x\right)^{\frac{1}{p}}<\infty\right\}, \\
L_{v}^{\infty}:=\left\{f \in \mathfrak{M}:\|f\|_{L_{v}^{\infty}}:=\underset{x \geq 0}{\operatorname{ess} \sup } v(x)|f(x)|<\infty\right\} .
\end{gathered}
$$

Let $0<q \leq \infty$ and $w \in \mathfrak{M}^{+}$. We consider quasilinear operators on $\mathfrak{M}^{+}$of the form

$$
\begin{aligned}
& (T f)(x)=\left(\int_{x}^{\infty} w(y)\left(\int_{0}^{y} k(y, z) f(z) d z\right)^{q} d y\right)^{\frac{1}{q}} \\
& (\mathscr{T} f)(x)=\left(\int_{0}^{x} w(y)\left(\int_{y}^{\infty} k(z, y) f(z) d z\right)^{q} d y\right)^{\frac{1}{q}}, \\
& (S f)(x)=\left(\int_{x}^{\infty} w(y)\left(\int_{y}^{\infty} k(z, y) f(z) d z\right)^{q} d y\right)^{\frac{1}{q}}, \\
& (\mathscr{S} f)(x)=\left(\int_{0}^{x} w(y)\left(\int_{0}^{y} k(y, z) f(z) d z\right)^{q} d y\right)^{\frac{1}{q}}
\end{aligned}
$$

and

$$
(\mathbf{T} f)(x)=\left(\int_{x}^{\infty} k(y, x) w(y)\left(\int_{0}^{y} f(z) d z\right)^{q} d y\right)^{\frac{1}{q}}
$$

${ }^{*}$ The research work of D.V. Prokhorov and V.D. Stepanov was financially supported by the Russian Scientific Fund (Project 14-11-00443). 


$$
\begin{aligned}
& (\mathfrak{T} f)(x)=\left(\int_{0}^{x} k(x, y) w(y)\left(\int_{y}^{\infty} f(z) d z\right)^{q} d y\right)^{\frac{1}{q}}, \\
& (\mathbf{S} f)(x)=\left(\int_{x}^{\infty} k(y, x) w(y)\left(\int_{y}^{\infty} f(z) d z\right)^{q} d y\right)^{\frac{1}{q}}, \\
& (\mathfrak{S} f)(x)=\left(\int_{0}^{x} k(x, y) w(y)\left(\int_{0}^{y} f(z) d z\right)^{q} d y\right)^{\frac{1}{q}},
\end{aligned}
$$

where $k(x, y) \geq 0$ is a measurable kernel and the right hand sides are to replace by essential supremums

$$
\begin{aligned}
& (T f)(x)=\underset{y \geq x}{\operatorname{essup}} w(y) \int_{0}^{y} k(y, z) f(z) d z, \\
& (\mathbf{T} f)(x)=\underset{y \geq x}{\operatorname{essipup}} k(y, x) w(y) \int_{0}^{y} f(z) d z,
\end{aligned}
$$

and similarly for the others, when $q=\infty$.

Let $u, v, w \in \mathfrak{M}^{+}$be weights, $1 \leq p \leq \infty, 0<r \leq \infty$. Our aim is to characterize the weighted inequalities

$$
\begin{aligned}
\|T f\|_{L_{u}^{r}} & \leq C_{T}\|f\|_{L_{v}^{p}}, f \in \mathfrak{M}^{+}, \\
\|\mathscr{T} f\|_{L_{u}^{r}} & \leq C_{\mathscr{T}}\|f\|_{L_{v}^{p}}, f \in \mathfrak{M}^{+}, \\
\|S f\|_{L_{u}^{r}} & \leq C_{S}\|f\|_{L_{v}^{p}}, f \in \mathfrak{M}^{+}, \\
\|\mathscr{S} f\|_{L_{u}^{r}} & \leq C_{\mathscr{S}}\|f\|_{L_{v}^{p}}, f \in \mathfrak{M}^{+}
\end{aligned}
$$

and

$$
\begin{aligned}
\|\mathbf{T} f\|_{L_{u}^{r}} & \leq C_{\mathbf{T}}\|f\|_{L_{v}^{p}}, f \in \mathfrak{M}^{+}, \\
\|\mathfrak{T} f\|_{L_{u}^{r}} & \leq C_{\mathfrak{T}}\|f\|_{L_{v}^{p}}, f \in \mathfrak{M}^{+}, \\
\|\mathbf{S} f\|_{L_{u}^{r}} & \leq C_{\mathbf{S}}\|f\|_{L_{v}^{p}}, f \in \mathfrak{M}^{+}, \\
\|\mathfrak{S} f\|_{L_{u}^{r}} & \leq C_{\mathfrak{S}}\|f\|_{L_{v}^{p}}, f \in \mathfrak{M}^{+},
\end{aligned}
$$

where a Borel function $k(x, y) \geq 0$ on $[0, \infty)^{2}$ satisfies Oinarov's condition: $k(x, y)=0$ if $x<y$, and there is a constant $D \geq 1$ independent of $x \geq z \geq y \geq 0$ such that

$$
\frac{1}{D}(k(x, z)+k(z, y)) \leq k(x, y) \leq D(k(x, z)+k(z, y))
$$

and the constants $C_{T}$ and others are taken as the least possible. If $q=r<\infty$ these inequalities are reduced to the generalized Hardy-type inequalities which were well studied see, for instance, [2], [21], [36] with further extensions and improvements in [19], [20], [22], [23], [26], [40], [41] and others. The case $q=\infty$ is closely related to recently initiated studies of supremum operators [12], [13], [24], [25], [27], [29], [37]. If $k(x, y) \equiv 1$ the inequality (1.4) plays an important role in analysis on the Morry-type spaces (see, [3], [4], [5], [6], [7]. In particular, for some parameters $p, q, r$ this case of (1.2) was solved in [16], [17] and (1.4) in [7]. Complete solution of this case is given in [30], [31].

By a new method we characterize the inequalities (1.1)-(1.8) with a kernel $k(x, y)$ satisfying (1.9) for all parameters $1 \leq p \leq \infty, 0<r \leq \infty, 0<q \leq \infty$. The cases $p=\infty$ and $r=\infty$ are trivial and the interval $0<p<1$ is excluded because in this case it can be shown that if, say, $C_{T}<\infty$, then $C_{T}=0$ (see [28], Theorem 2 for details). 
Sections 2 and 3 are devoted to the study of (1.1)-(1.4) and sections 4 and 5 to (1.5)(1.8). It is interesting to observe that the second part is partially based on the first. In the last section 6 we illustrate our results by a solution of well known problem on a sharp characterization of the $\Gamma^{p}(v) \rightarrow \Gamma^{q}(w)$ boundedness of the Hardy-Littlewood maximal operator for all $0<p, q<\infty$ including the most difficult cases missed in [10] and [33].

We use signs $:=$ and $=$ : for determining new quantities and $\mathbb{Z}$ for the set of all integers. For positive functionals $F$ and $G$ we write $F \lesssim G$, if $F \leq c G$ with some positive constant $c$, which depends only on irrelevant parameters. $F \approx G$ means $F \lesssim G \lesssim F$ or $F=c G$. $\chi_{E}$ denotes the characteristic function (indicator) of a set $E$. Uncertainties of the form $0 \cdot \infty, \frac{\infty}{\infty}$ and $\frac{0}{0}$ are taken to be zero. $\square$ stands for the end of a proof.

\section{Operators $T$ and $S$}

Suppose for simplicity that $\int_{0}^{t} u<\infty$ for all $t>0$ and define the functions $\sigma:[0, \infty) \rightarrow[0, \infty]$, $\sigma^{-1}:[0, \infty) \rightarrow[0, \infty)$ by (here inf $\varnothing=\infty$ )

$$
\sigma(x):=\inf \left\{y>0: \int_{0}^{y} u \geq 2 \int_{0}^{x} u\right\}, \quad \sigma^{-1}(x):=\inf \left\{y>0: \int_{0}^{y} u \geq \frac{1}{2} \int_{0}^{x} u\right\} .
$$

The functions $\sigma$ and $\sigma^{-1}$ are increasing and from the continuity of an integral with respect to an upper limit it follows for any $x \in[0, \infty)$ that $\int_{0}^{\sigma^{-1}(x)} u=\frac{1}{2} \int_{0}^{x} u$ and if $\sigma(x)<\infty$, then $\int_{0}^{\sigma(x)} u=2 \int_{0}^{x} u$.

Let $\sigma^{m}, m \in \mathbb{N}$ be a composition of $m$ functions $\sigma$ and similar for $\sigma^{-m}$.

For $0<c<d \leq \infty$ and $f \in \mathfrak{M}^{+}$we put

$$
\begin{gathered}
\left(H_{c, d} f\right)(x):=\chi_{[c, d)}(x) \int_{\sigma^{-1}(c)}^{x} k(x, z) f(z) d z, \\
\left(H_{c} f\right)(x):=\chi_{[c, \infty)}(x) \int_{0}^{x} k(x, z) f(z) d z .
\end{gathered}
$$

Theorem 2.1. Let $1 \leq p<\infty, 0<r<\infty, 0<q \leq \infty, \frac{1}{s}:=\left(\frac{1}{r}-\frac{1}{p}\right)_{+}$. For validity of the inequality (1.1) it is necessary and sufficient that the inequalities

$$
\begin{aligned}
& \left(\int_{0}^{\infty} u(x)\left(\int_{x}^{\infty} w\right)^{\frac{r}{q}}\left(\int_{0}^{x} k(x, z) f(z) d z\right)^{r} d x\right)^{\frac{1}{r}} \leq A_{0}\|f\|_{L_{v}^{p}}, \\
& \left(\int_{0}^{\infty} u(x)\left(\int_{x}^{\infty}[k(z, x)]^{q} w(z) d z\right)^{\frac{r}{q}}\left(\int_{0}^{x} f\right)^{r} d x\right)^{\frac{1}{r}} \leq A_{1}\|f\|_{L_{v}^{p}},
\end{aligned}
$$

if $q<\infty$ or

$$
\begin{aligned}
& \left(\int_{0}^{\infty} u(x)\left[\operatorname{essup}_{y \geq x} w(y)\right]^{r}\left(\int_{0}^{x} k(x, z) f(z) d z\right)^{r} d x\right)^{\frac{1}{r}} \leq A_{0}\|f\|_{L_{v}^{p}} \\
& \left.\left(\int_{0}^{\infty} u(x) \underset{y \geq x}{\operatorname{ess} \sup }[w(y) k(y, x)]\right]^{r}\left(\int_{0}^{x} f\right)^{r} d x\right)^{\frac{1}{r}} \leq A_{1}\|f\|_{L_{v}^{p}}
\end{aligned}
$$


for $q=\infty$ hold for all $f \in \mathfrak{M}^{+}$and the constant

$$
A_{2}:= \begin{cases}\sup _{t \in(0, \infty)}\left(\int_{0}^{t} u\right)^{\frac{1}{r}}\left\|H_{t}\right\|_{L_{v}^{p} \rightarrow L_{w}^{q},} & p \leq r, \\ \left(\int_{0}^{\infty} u(x)\left(\int_{0}^{x} u\right)^{\frac{s}{p}}\left\|H_{\sigma^{-1}(x), \sigma(x)}\right\|_{L_{v}^{p} \rightarrow L_{w}^{q}}^{s} d x\right)^{\frac{1}{s}}, & r<p\end{cases}
$$

is finite. Moreover, $C_{T} \approx A_{0}+A_{1}+A_{2}$.

Proof. Let $n_{0} \in \mathbb{Z}$ be such an integer that $2^{n_{0}}<\int_{0}^{\infty} u$. Put

$$
\begin{aligned}
a_{n_{0}} & :=\inf \left\{y>0: \int_{0}^{y} u \geq 2^{n_{0}}\right\}, \\
a_{n+1} & :=\sigma\left(a_{n}\right) \text { for } n \geq n_{0}, \\
a_{n-1} & :=\sigma^{-1}\left(a_{n}\right) \text { for } n \leq n_{0} .
\end{aligned}
$$

Denote $N:=\sup \left\{n \in \mathbb{Z}: a_{n}<\infty\right\}$. If $N<\infty$ we put $a_{N+1}:=\infty$. Observe, that $a_{n-1}=$ $\sigma^{-1}\left(a_{n}\right)$ and $\sigma\left(a_{n}\right)=a_{n+1}$ for all $n \leq N$.

We suppose first that $q<\infty$.

Sufficiency. Let $\Delta_{n}:=\left[a_{n}, a_{n+1}\right)$. Applying the condition (1.9) and the relation ( [10], Proposition 2.1)

$$
\sum_{n \in \mathbb{Z}} 2^{n}\left(\sum_{i \geq n} \lambda_{i}\right)^{s} \approx \sum_{n \in \mathbb{Z}} 2^{n} \lambda_{n}^{s},
$$

which is valid for all sequences $\left\{\lambda_{n}\right\}$ of non-negative numbers and any $s>0$, we have

$$
\begin{aligned}
\int_{0}^{\infty}[T f]^{r} u & =\sum_{n \leq N} \int_{\Delta_{n}}[T f]^{r} u \approx \sum_{n \leq N} 2^{n}\left(\int_{a_{n}}^{\infty} w(y)\left(\int_{0}^{y} k(y, z) f(z) d z\right)^{q} d y\right)^{\frac{r}{q}} \\
& \approx \sum_{n \leq N} 2^{n}\left(\int_{\Delta_{n}} w(y)\left(\int_{0}^{y} k(y, z) f(z) d z\right)^{q} d y\right)^{\frac{r}{q}} \\
& \approx \sum_{n \leq N} 2^{n}\left(\int_{\Delta_{n}} w(y)\left(\int_{a_{n-1}}^{y} k(y, z) f(z) d z\right)^{q} d y\right)^{\frac{r}{q}} \\
& +\sum_{n \leq N} 2^{n}\left(\int_{\Delta_{n}} w(y)\left(\int_{0}^{a_{n-1}} k(y, z) f(z) d z\right)^{q} d y\right)^{\frac{r}{q}}=: J_{1}^{r}+J_{2}^{r} .
\end{aligned}
$$

Since $k(y, z) \approx k(y, x)+k(x, z)$ for $y \in \Delta_{n}, x \in \Delta_{n-1}, z \in\left(0, a_{n-1}\right)$, then $J_{2}^{r}$ is estimated as 
follows

$$
\begin{aligned}
& J_{2}^{r} \approx \sum_{n \leq N} \int_{a_{n-1}}^{a_{n}} u(x) d x\left(\int_{\Delta_{n}} w(y)\left(\int_{0}^{a_{n-1}} k(y, z) f(z) d z\right)^{q} d y\right)^{\frac{r}{q}} \\
& \approx \sum_{n \leq N} \int_{a_{n-1}}^{a_{n}} u(x)\left(\int_{a_{n}}^{a_{n+1}} w(y)[k(y, x)]^{q} d y\right)^{\frac{r}{q}} d x\left(\int_{0}^{a_{n-1}} f\right)^{r} \\
& +\sum_{n \leq N} \int_{a_{n-1}}^{a_{n}} u(x)\left(\int_{0}^{a_{n-1}} k(x, z) f(z) d z\right)^{r} d x\left(\int_{\Delta_{n}} w\right)^{\frac{r}{q}} \\
& \lesssim \sum_{n \leq N} \int_{a_{n-1}}^{a_{n}} u(x)\left(\int_{x}^{\infty} w(y)[k(y, x)]^{q} d y\right)^{\frac{r}{q}}\left(\int_{0}^{x} f\right)^{r} d x \\
& +\sum_{n \leq N} \int_{a_{n-1}}^{a_{n}} u(x)\left(\int_{x}^{\infty} w\right)^{\frac{r}{q}}\left(\int_{0}^{x} k(x, z) f(z) d z\right)^{r} d x \\
& \lesssim\left(A_{1}^{r}+A_{0}^{r}\right)\|f\|_{L_{v}^{p}}^{r} .
\end{aligned}
$$

For an upper bound of $J_{1}^{r}$ we write

$$
\begin{aligned}
J_{1}^{r} & \approx \sum_{n \leq N} 2^{n}\left\|H_{a_{n}, a_{n+1}} f\right\|_{L_{w}^{q}}^{r} \\
& \lesssim \sum_{n \leq N}\left(\int_{a_{n-1}}^{a_{n}} u\right)\left\|H_{a_{n}, a_{n+1}}\right\|_{L_{v}^{p} \rightarrow L_{w}^{q}}^{r}\left(\int_{a_{n-1}}^{a_{n+1}} f^{p} v\right)^{\frac{r}{p}} .
\end{aligned}
$$

If $p \leq r$ we apply Jensen's inequality and get

$$
J_{1} \lesssim \sup _{n \leq N}\left(\int_{a_{n-1}}^{a_{n}} u\right)^{\frac{1}{r}}\left\|H_{a_{n}, a_{n+1}}\right\|_{L_{v}^{p} \rightarrow L_{w}^{q}}\|f\|_{L_{v}^{p}} \leq A_{2}\|f\|_{L_{v}^{p}}
$$

If $r<p$ we apply Hölder's inequality with the exponents $\frac{s}{r}$ and $\frac{p}{r}$ and obtain

$$
\begin{aligned}
J_{1}^{s} & \lesssim \sum_{n \leq N}\left(\int_{a_{n}}^{a_{n+1}} u\right)^{\frac{s}{r}}\left\|H_{a_{n}, a_{n+1}}\right\|_{L_{v}^{p} \rightarrow L_{w}^{q}}^{s}\|f\|_{L_{v}^{p}}^{s} \\
& \lesssim \sum_{n \leq N}\left(\int_{a_{n}}^{a_{n+1}} u\right)\left(\int_{0}^{a_{n}} u\right)^{\frac{s}{p}}\left\|H_{\sigma^{-1}\left(a_{n+1}\right), \sigma\left(a_{n}\right)}\right\|_{L_{v}^{p} \rightarrow L_{w}^{q}}^{s}\|f\|_{L_{v}^{p}}^{s} \\
& \leq \sum_{n \leq N}\left(\int_{a_{n}}^{a_{n+1}} u(x)\left(\int_{0}^{x} u\right)^{\frac{s}{p}}\left\|H_{\sigma^{-1}(x), \sigma(x)}\right\|_{L_{v}^{p} \rightarrow L_{w}^{q}}^{s} d x\right)\|f\|_{L_{v}^{p}}^{s} \\
& \leq A_{2}^{s}\|f\|_{L_{v}^{p} .}^{s}
\end{aligned}
$$

Thus,

$$
\|T f\|_{L_{u}^{r}} \lesssim\left(A_{0}+A_{1}+A_{2}\right)\|f\|_{L_{v}^{p}}
$$

and the upper bound $C_{T} \lesssim A_{0}+A_{1}+A_{2}$ is proved. 
Necessity. Since

$$
\begin{aligned}
(T f)(x) & \geq\left(\int_{x}^{\infty} w(y)\left(\int_{0}^{x} k(y, z) f(z) d z\right)^{q} d y\right)^{\frac{1}{q}} \\
& \gtrsim\left(\int_{x}^{\infty} w\right)^{\frac{1}{q}} \int_{0}^{x} k(x, z) f(z) d z
\end{aligned}
$$

the inequality (1.1) implies (2.1) and $C_{T} \gtrsim A_{0}$. Moreover,

$$
\begin{aligned}
(T f)(x) & \geq\left(\int_{x}^{\infty} w(y)\left(\int_{0}^{x} k(y, z) f(z) d z\right)^{q} d y\right)^{\frac{1}{q}} \\
& \gtrsim\left(\int_{x}^{\infty}[k(y, x)]^{q} w(y) d y\right)^{\frac{1}{q}} \int_{0}^{x} f .
\end{aligned}
$$

Then (1.1) implies (2.2) and $C_{T} \gtrsim A_{1}$. It follows from (1.1) that

$$
C_{T}\|f\|_{L_{v}^{p}} \geq\left(\int_{0}^{t} u\right)^{\frac{1}{r}}\left\|H_{t} f\right\|_{L_{w}^{q}}, f \in \mathfrak{M}^{+}
$$

for any $t \in(0, \infty)$. Hence,

$$
C_{T} \geq \sup _{t \in(0, \infty)}\left(\int_{0}^{t} u\right)^{\frac{1}{r}}\left\|H_{t}\right\|_{L_{v}^{p} \rightarrow L_{w}^{q}}
$$

and the lower bound $C_{T} \gtrsim A_{2}$ is proved for $p \leq r$. Now, let $r<p$. We have

$$
\begin{aligned}
A_{2}^{s} & =\int_{0}^{\infty} u(x)\left(\int_{0}^{x} u\right)^{\frac{s}{p}}\left\|H_{\sigma^{-1}(x), \sigma(x)}\right\|_{L_{v}^{p} \rightarrow L_{w}^{q}}^{s} d x \\
& =\sum_{n \leq N} \int_{a_{n}}^{a_{n+1}} u(x)\left(\int_{0}^{x} u\right)^{\frac{s}{p}}\left\|H_{\sigma^{-1}(x), \sigma(x)}\right\|_{L_{v}^{p} \rightarrow L_{w}^{q}}^{s} d x \\
& \leq \sum_{n \leq N}\left(\int_{a_{n}}^{a_{n+1}} u\right)\left(\int_{0}^{a_{n+1}} u\right)^{\frac{s}{p}}\left\|H_{\sigma^{-1}\left(a_{n}\right), \sigma\left(a_{n+1}\right)}\right\|_{L_{v}^{p} \rightarrow L_{w}^{q}}^{s} \\
& \approx \sum_{n \leq N}\left(2^{n}\right)^{\frac{s}{r}}\left\|H_{a_{n-1}, a_{n+2}}\right\|_{L_{v}^{p} \rightarrow L_{w}^{q}}^{s}=: \bar{A}_{2}^{s} .
\end{aligned}
$$

Let $\theta \in(0,1)$ be arbitrary. For all $n \leq N$ there is $f_{n} \in \mathfrak{M}^{+}$such that $\operatorname{supp} f_{n} \subset\left[a_{n-2}, a_{n+2}\right]$, $\left\|f_{n}\right\|_{L_{v}^{p}}=1$ and

$$
\left\|H_{a_{n-1}, a_{n+2}} f_{n}\right\|_{L_{w}^{q}} \geq \theta\left\|H_{a_{n-1}, a_{n+2}}\right\|_{L_{v}^{p} \rightarrow L_{w}^{q}} .
$$

Put

$$
g_{n}:=\left(2^{n}\right)^{\frac{s}{p r}}\left\|H_{a_{n-1}, a_{n+2}}\right\|_{L_{v}^{p} \rightarrow L_{w}^{q}}^{\frac{s}{p}} f_{n}, \quad g:=\sum_{n \leq N} g_{n} .
$$


We find

$$
\begin{aligned}
\|g\|_{L_{v}^{p}}^{p} & =\sum_{j \leq N} \int_{a_{j}}^{a_{j+1}}\left(\sum_{n \leq N} g_{n}(x)\right)^{p} v(x) d x \\
& =\sum_{j \leq N} \int_{a_{j}}^{a_{j+1}}\left(\sum_{n=j-1}^{j+2} g_{n}(x)\right)^{p} v(x) d x \\
& \lesssim \sum_{j \leq N} \int_{a_{j-2}}^{a_{j+2}} g_{j}(x)^{p} v(x) d x \\
& =\sum_{j \leq N}\left(2^{j}\right)^{\frac{s}{r}}\left\|H_{a_{j-1}, a_{j+2}}\right\|_{L_{v}^{p} \rightarrow L_{w}^{q}}^{s}=\bar{A}_{2}^{s} .
\end{aligned}
$$

Finally, applying (1.1)

$$
\begin{aligned}
C_{T}^{r} \bar{A}_{2}^{\frac{s r}{p}} & \gtrsim C_{T}^{r}\|g\|_{L_{v}^{p}}^{r} \geq \int_{0}^{\infty}[T g]^{r} u \geq \sum_{n \leq N} \int_{a_{n-2}}^{a_{n-1}}[T g]^{r} u \\
& \geq \sum_{n \leq N}\left(\int_{a_{n-2}}^{a_{n-1}} u\right)\left\|H_{a_{n-1}, a_{n+2}} g\right\|_{L_{w}^{q}}^{r} \gtrsim \sum_{n \leq N} 2^{n}\left\|H_{a_{n-1}, a_{n+2}} g_{n}\right\|_{L_{w}^{q}}^{r} \\
& =\sum_{n \leq N}\left(2^{n}\right)^{\frac{s}{r}}\left\|H_{a_{n-1}, a_{n+2}}\right\|_{L_{v}^{p} \rightarrow L_{w}^{q}}^{\frac{s r}{p}}\left\|H_{a_{n-1}, a_{n+2}} f_{n}\right\|_{L_{w}^{q}}^{r} \\
& \geq \theta^{r} \sum_{n \leq N}\left(2^{n}\right)^{\frac{s}{r}}\left\|H_{a_{n-1}, a_{n+2}}\right\|_{L_{v}^{p} \rightarrow L_{w}^{q}}^{s} \gtrsim \theta^{r} \bar{A}_{2}^{s} .
\end{aligned}
$$

Thus, $C_{T} \gtrsim \theta \bar{A}_{2}$. Hence, $C_{T} \gtrsim \theta A_{2}$ and the required lower bound $C_{T} \gtrsim A_{0}+A_{1}+A_{2}$ follows.

The case $q=\infty$ is treated similarly with only replacement of (2.6) by a trivial modification

$$
\sum_{n \in \mathbb{Z}} 2^{n}\left(\sup _{i \geq n} \lambda_{i}\right)^{s} \approx \sum_{n \in \mathbb{Z}} 2^{n} \lambda_{n}^{s} .
$$

Remark 2.2. For $p=\infty$ we have

$$
C_{T}=\left\|T\left(\frac{1}{v}\right)\right\|_{L_{u}^{r}}
$$

and for $r=\infty$

$$
C_{T}=\sup _{t \geq 0} U(t)\left\|H_{t}\right\|_{L_{v}^{p} \rightarrow L_{w}^{q}}
$$

where $U(t):=\operatorname{esssup}_{0 \leq x \leq t} u(x)$.

Now, for $0<c<d \leq \infty$ and $f \in \mathfrak{M}^{+}$we put

$$
\begin{aligned}
\left(H_{c, d}^{*} f\right)(x) & :=\chi_{[c, d)}(x) \int_{x}^{\sigma(d)} k(z, x) f(z) d z \\
\left(H_{c}^{*} f\right)(x) & :=\chi_{[c, \infty)}(x) \int_{x}^{\infty} k(z, x) f(z) d z .
\end{aligned}
$$


Theorem 2.3. Let $1 \leq p<\infty, 0<r<\infty, 0<q \leq \infty, \frac{1}{s}:=\left(\frac{1}{r}-\frac{1}{p}\right)_{+}$. Then the inequality (1.3) is fulfilled if and only if the inequalities

$$
\begin{aligned}
& \left(\int_{0}^{\infty} u(x)\left(\int_{x}^{\sigma^{2}(x)} w\right)^{\frac{r}{q}}\left(\int_{\sigma^{2}(x)}^{\infty} k\left(z, \sigma^{2}(x)\right) f(z) d z\right)^{r} d x\right)^{\frac{1}{r}} \leq \mathbb{A}_{0}\|f\|_{L_{v}^{p}} \\
& \left(\int_{0}^{\infty} u(x)\left(\int_{x}^{\sigma^{2}(x)}\left[k\left(\sigma^{2}(x), z\right)\right]^{q} w(z) d z\right)^{\frac{r}{q}}\left(\int_{\sigma^{2}(x)}^{\infty} f\right)^{r} d x\right)^{\frac{1}{r}} \leq \mathbb{A}_{1}\|f\|_{L_{v}^{p}}
\end{aligned}
$$

if $q<\infty$ or

$$
\begin{aligned}
& \left(\int_{0}^{\infty} u(x)\left[\operatorname{essup}_{y \in\left(x, \sigma^{2}(x)\right)} w(y)\right]^{r}\left(\int_{\sigma^{2}(x)}^{\infty} k\left(z, \sigma^{2}(x)\right) f(z) d z\right)^{r} d x\right)^{\frac{1}{r}} \leq \mathbb{A}_{0}\|f\|_{L_{v}^{p}}, \\
& \left(\int_{0}^{\infty} u(x)\left[{\operatorname{ess} \sup _{y \in\left(x, \sigma^{2}(x)\right)}}_{\operatorname{en}}\left[w(y) k\left(\sigma^{2}(x), y\right)\right]^{r}\left(\int_{\sigma^{2}(x)}^{\infty} f\right)^{r} d x\right)^{\frac{1}{r}} \leq \mathbb{A}_{1}\|f\|_{L_{v}^{p}}\right.
\end{aligned}
$$

for $q=\infty$ hold for all $f \in \mathfrak{M}^{+}$and the constant

$$
\mathbb{A}_{2}:= \begin{cases}\sup _{t \in(0, \infty)}\left(\int_{0}^{t} u\right)^{\frac{1}{r}}\left\|H_{t}^{*}\right\|_{L_{v}^{p} \rightarrow L_{w}^{q},} & p \leq r, \\ \left(\int_{0}^{\infty} u(x)\left(\int_{0}^{x} u\right)^{\frac{s}{p}}\left\|H_{\sigma^{-1}(x), \sigma(x)}^{*}\right\|_{L_{v}^{p} \rightarrow L_{w}^{q}}^{s} d x\right)^{\frac{1}{s}}, & r<p\end{cases}
$$

is finite. Moreover, $C_{S} \approx \mathbb{A}_{0}+\mathbb{A}_{1}+\mathbb{A}_{2}$.

Proof. Let the sequence $\left\{a_{n}\right\}$ be the same as in the proof of Theorem 2.1 and $q<\infty$.

Sufficiency. We have

$$
\begin{aligned}
\int_{0}^{\infty}[S f]^{r} u & =\sum_{n \leq N} \int_{\Delta_{n}}[S f]^{r} u \approx \sum_{n \leq N} 2^{n}\left(\int_{a_{n}}^{\infty} w(y)\left(\int_{y}^{\infty} k(z, y) f(z) d z\right)^{q} d y\right)^{\frac{r}{q}} \\
& \approx \sum_{n \leq N} 2^{n}\left(\int_{\Delta_{n}} w(y)\left(\int_{y}^{\infty} k(z, y) f(z) d z\right)^{q} d y\right)^{\frac{r}{q}} \\
& \approx \sum_{n \leq N} 2^{n}\left(\int_{\Delta_{n}} w(y)\left(\int_{y}^{a_{n+2}} k(z, y) f(z) d z\right)^{q} d y\right)^{\frac{r}{q}} \\
& +\sum_{n \leq N} 2^{n}\left(\int_{\Delta_{n}} w(y)\left(\int_{a_{n+2}}^{\infty} k(z, y) f(z) d z\right)^{q} d y\right)^{\frac{r}{q}}=: I_{1}^{r}+I_{2}^{r} .
\end{aligned}
$$

Since for $y \in \Delta_{n}, x \in \Delta_{n-1}, z \in\left(a_{n+2}, \infty\right)$ it holds $k(z, y) \approx k\left(z, \sigma^{2}(x)\right)+k\left(\sigma^{2}(x), y\right)$, then the term $I_{2}^{r}$ is estimated as follows 


$$
\begin{aligned}
I_{2}^{r} & \lesssim \sum_{n \leq N} \int_{a_{n-1}}^{a_{n}} u(x)\left(\int_{\Delta_{n}} w(y)\left(\int_{a_{n+2}}^{\infty} k(z, y) f(z) d z\right)^{q} d y\right)^{\frac{r}{q}} d x \\
& \lesssim \sum_{n \leq N} \int_{a_{n-1}}^{a_{n}} u(x)\left(\int_{\Delta_{n}} w\right)^{\frac{r}{q}}\left(\int_{\sigma^{2}(x)}^{\infty} k\left(z, \sigma^{2}(x)\right) f(z) d z\right)^{r} d x \\
& +\sum_{n \leq N} \int_{a_{n-1}}^{a_{n}} u(x)\left(\int_{\Delta_{n}}\left[k\left(\sigma^{2}(x), y\right)\right]^{q} w(y) d y\right)^{\frac{r}{q}}\left(\int_{\sigma^{2}(x)}^{\infty} f\right)^{r} d x \\
& \lesssim \sum_{n \leq N} \int_{a_{n-1}}^{a_{n}} u(x)\left(\int_{x}^{\sigma^{2}(x)} w\right)^{\frac{r}{q}}\left(\int_{\sigma^{2}(x)}^{\infty} k\left(z, \sigma^{2}(x)\right) f(z) d z\right)^{r} d x \\
& +\sum_{n \leq N} \int_{a_{n-1}}^{a_{n}} u(x)\left(\int_{x}^{\sigma^{2}(x)}\left[k\left(\sigma^{2}(x), y\right)\right]^{q} w(y) d y\right)^{\frac{r}{q}}\left(\int_{\sigma^{2}(x)}^{\infty} f\right)^{r} d x \\
& \leq\left(\mathbb{A}_{0}^{r}+\mathbb{A}_{1}^{r}\right)\left(\int_{0}^{\infty} f^{p} v\right)^{\frac{r}{p}} .
\end{aligned}
$$

To estimate $I_{1}^{r}$ we write

$$
I_{1}^{r} \lesssim \sum_{n \leq N}\left(\int_{a_{n-1}}^{a_{n}} u\right)\left\|H_{a_{n}, a_{n+1}}^{*}\right\|_{L_{v}^{p} \rightarrow L_{w}^{q}}^{r}\left(\int_{a_{n}}^{a_{n+2}} f^{p} v\right)^{\frac{r}{p}} .
$$

If $p \leq r$, by Jensen's inequality

$$
I_{1} \lesssim \sup _{n \leq N}\left(\int_{a_{n-1}}^{a_{n}} u\right)^{\frac{1}{r}}\left\|H_{a_{n}, a_{n+1}}^{*}\right\|\left\|_{L_{v}^{p} \rightarrow L_{w}^{q}}\right\| f\left\|_{L_{v}^{p}} \leq \mathbb{A}_{2}\right\| f \|_{L_{v}^{p}} .
$$

If $r<p$, applying Hölder's inequality with exponents $\frac{s}{r}$ and $\frac{p}{r}$ similar to the proof of Theorem 2.1, we find

$$
\begin{aligned}
I_{1}^{s} & \lesssim \sum_{n \leq N}\left(\int_{a_{n}}^{a_{n+1}} u\right)^{\frac{s}{r}}\left\|H_{a_{n}, a_{n+1}}^{*}\right\|_{L_{v}^{p} \rightarrow L_{w}^{q}}^{s}\|f\|_{L_{v}^{p}}^{s} \\
& \lesssim \sum_{n \leq N}\left(\int_{a_{n}}^{a_{n+1}} u(x)\left(\int_{0}^{x} u\right)^{\frac{s}{p}}\left\|H_{\sigma^{-1}(x), \sigma(x)}^{*}\right\|_{L_{v}^{p} \rightarrow L_{w}^{q}}^{s} d x\right)\|f\|_{L_{v}^{p}}^{s} \\
& \leq \mathbb{A}_{2}^{s}\|f\|_{L_{v}^{p}}^{s} .
\end{aligned}
$$

Thus, $C_{S} \lesssim \mathbb{A}_{0}+\mathbb{A}_{1}+\mathbb{A}_{2}$.

Necessity. Since

$$
(S f)(x) \gtrsim\left\|\chi_{\left[x, \sigma^{2}(x)\right)}\right\|_{L_{w}^{q}} \int_{\sigma^{2}(x)}^{\infty} k\left(z, \sigma^{2}(x)\right) f(z) d z,
$$


then (1.3) implies (2.10) and $C_{S} \gtrsim \mathbb{A}_{0}$. Also,

$$
\begin{aligned}
(S f)(x) & \geq\left(\int_{x}^{\sigma^{2}(x)} w(y)\left(\int_{\sigma^{2}(x)}^{\infty} k(z, y) f(z) d z\right)^{q}\right)^{\frac{1}{q}} \\
& \gtrsim\left(\int_{x}^{\sigma^{2}(x)} w(y) k\left(\sigma^{2}(x), y\right) d y\right)^{\frac{1}{q}} \int_{\sigma^{2}(x)}^{\infty} f .
\end{aligned}
$$

Therefore, (1.3) implies (2.11) and $C_{S} \gtrsim \mathbb{A}_{1}$.

Now, let $t \in(0, \infty)$ be fixed. It follows from (1.3)

$$
C_{S}\|f\|_{L_{v}^{p}} \geq\left(\int_{0}^{t} u\right)^{\frac{1}{r}}\left\|H_{t}^{*} f\right\|_{L_{w}^{q}}, f \in \mathfrak{M}^{+} .
$$

Hence,

$$
C_{S} \geq \sup _{t \in(0, \infty)}\left(\int_{0}^{t} u\right)^{\frac{1}{r}}\left\|H_{t}^{*}\right\|_{L_{v}^{p} \rightarrow L_{w}^{q}}
$$

and $C_{S} \gtrsim \mathbb{A}_{2}$ for $p \leq r$ is shown.

Now, let $r<p$. As in the proof of Theorem 2.1 we find

$$
\begin{aligned}
\mathbb{A}_{2}^{s} & =\int_{0}^{\infty} u(x)\left(\int_{0}^{x} u\right)^{\frac{s}{p}}\left\|H_{\sigma^{-1}(x), \sigma(x)}^{*}\right\|_{L_{v}^{p} \rightarrow L_{w}^{q}}^{s} d x \\
& \lesssim \sum_{n \leq N}\left(2^{n}\right)^{\frac{s}{r}}\left\|H_{a_{n-1}, a_{n+2}}^{*}\right\|_{L_{v}^{p} \rightarrow L_{w}^{q}}^{s}
\end{aligned}
$$

Let $\theta \in(0,1)$ be arbitrary. For all $n \leq N$ there is $f_{n} \in \mathfrak{M}^{+}$such that $\operatorname{supp} f_{n} \subset\left[a_{n-1}, a_{n+3}\right]$, $\left\|f_{n}\right\|_{L_{v}^{p}}=1$ and

$$
\left\|H_{a_{n-1}, a_{n+2}}^{*} f_{n}\right\|_{L_{w}^{q}} \geq \theta\left\|H_{a_{n-1}, a_{n+2}}^{*}\right\|_{L_{v}^{p} \rightarrow L_{w}^{q}} .
$$

Put

$$
g_{n}:=\left(2^{n}\right)^{\frac{s}{p r}}\left\|H_{a_{n-1}, a_{n+2}}^{*}\right\|_{L_{v}^{p} \rightarrow L_{w}^{q}}^{\frac{s}{p}} f_{n}, \quad g:=\sum_{n \leq N} g_{n} .
$$

Then

$$
\begin{aligned}
\|g\|_{L_{v}^{p}}^{p} & =\sum_{j \leq N} \int_{a_{j}}^{a_{j+1}}\left(\sum_{n \leq N} g_{n}(x)\right)^{p} v(x) d x \\
& =\sum_{j \leq N} \int_{a_{j}}^{a_{j+1}}\left(\sum_{n=j-2}^{j+1} g_{n}(x)\right)^{p} v(x) d x \\
& \lesssim \sum_{j \leq N} \int_{a_{j-1}}^{a_{j+3}} g_{j}(x)^{p} v(x) d x=\sum_{j \leq N}\left(2^{j}\right)^{\frac{s}{r}}\left\|H_{a_{j-1}, a_{j+2}}^{*}\right\|_{L_{v}^{p} \rightarrow L_{w}^{q}}^{s}
\end{aligned}
$$

Now,

$$
\int_{0}^{\infty}[S g]^{r} u \gtrsim \theta^{r} \sum_{n \leq N}\left(2^{n}\right)^{\frac{s}{r}}\left\|H_{a_{n-1}, a_{n+2}}^{*}\right\|_{L_{v}^{p} \rightarrow L_{w}^{q}}^{s}
$$

and we obtain $C_{S} \gtrsim \mathbb{A}_{2}$ for $r<p$ and $C_{S} \gtrsim \mathbb{A}_{0}+\mathbb{A}_{1}+\mathbb{A}_{2}$ similar to the proof of Theorem 2.1. The case $q=\infty$ is proved analogously. 
Remark 2.4. Precise characterization of the inequalities (2.1)-(2.4), (2.10)-(2.13), sharp estimates of the norms $\left\|H_{t}\right\|_{L_{v}^{p} \rightarrow L_{w}^{q}},\left\|H_{\sigma^{-1}(x), \sigma(x)}\right\|_{L_{v}^{p} \rightarrow L_{w}^{q}},\left\|H_{t}^{*}\right\|_{L_{v}^{p} \rightarrow L_{w}^{q}}$ and $\left\|H_{\sigma^{-1}(x), \sigma(x)}^{*}\right\|_{L_{v}^{p} \rightarrow L_{w}^{q}}$ are known and can be found (in various, but equivalent forms) by using, for instance, the results of [40], [41] and [26], where an integral form of criterion for the case $0<q<1$ was found.

Remark 2.5. For $p=\infty$ we have

$$
C_{S}=\left\|S\left(\frac{1}{v}\right)\right\|_{L_{u}^{r}}
$$

and for $r=\infty$

$$
C_{S}=\sup _{t \geq 0} U(t)\left\|H_{t}^{*}\right\|_{L_{v}^{p} \rightarrow L_{w}^{q}}
$$

where $U(t):=\underset{0 \leq x \leq t}{\operatorname{ess} \sup } u(x)$

\section{Operators $\mathscr{T}$ and $\mathscr{S}$}

For finding criteria for (1.2) and (1.4) we suppose that $0<\int_{t}^{\infty} u<\infty$ for all $t>0$ and define the functions $\zeta:[0, \infty) \rightarrow[0, \infty), \zeta^{-1}:[0, \infty) \rightarrow[0, \infty)$ by

$$
\begin{gathered}
\zeta(x):=\sup \left\{y>0: \int_{y}^{\infty} u \geq \frac{1}{2} \int_{x}^{\infty} u\right\}, \\
\zeta^{-1}(x):=\sup \left\{y>0: \int_{y}^{\infty} u \geq 2 \int_{x}^{\infty} u\right\},
\end{gathered}
$$

where $\sup \varnothing=0$. Let, also, $\zeta^{m}, m \in \mathbb{N}$ be a composition of $m$ functions $\zeta$ and similar for $\zeta^{-m}$. For $0 \leq c<d<\infty$ and $f \in \mathfrak{M}^{+}$put

$$
\begin{aligned}
\left(\mathscr{H}_{c, d} f\right)(x) & :=\chi_{(c, d]}(x) \int_{x}^{\zeta(d)} k(z, x) f(z) d z, \\
\left(\mathscr{H}_{d} f\right)(x) & :=\chi_{(0, d]}(x) \int_{x}^{\infty} k(z, x) f(z) d z, \\
\left(\mathscr{H}_{c, d}^{*} f\right)(x) & :=\chi_{(c, d]}(x) \int_{\zeta^{-1}(c)}^{x} k(x, z) f(z) d z, \\
\left(\mathscr{H}_{d}^{*} f\right)(x) & :=\chi_{(0, d]}(x) \int_{0}^{x} k(x, z) f(z) d z .
\end{aligned}
$$

Similar to the previous section we prove the following theorems.

Theorem 3.1. Let $1 \leq p<\infty, 0<r<\infty, 0<q \leq \infty, \frac{1}{s}:=\left(\frac{1}{r}-\frac{1}{p}\right)_{+}$. For validity of the inequality (1.2) it is necessary and sufficient that the inequalities

$$
\begin{aligned}
& \left(\int_{0}^{\infty} u(x)\left(\int_{0}^{x} w\right)^{\frac{r}{q}}\left(\int_{x}^{\infty} k(z, x) f(z) d z\right)^{r} d x\right)^{\frac{1}{r}} \leq \mathscr{A}_{0}\|f\|_{L_{v}^{p}}, \\
& \left(\int_{0}^{\infty} u(x)\left(\int_{0}^{x}[k(x, y)]^{q} w(y) d y\right)^{\frac{r}{q}}\left(\int_{x}^{\infty} f\right)^{r} d x\right)^{\frac{1}{r}} \leq \mathscr{A}_{1}\|f\|_{L_{v}^{p}},
\end{aligned}
$$


if $q<\infty$ or

$$
\begin{aligned}
& \left(\int_{0}^{\infty} u(x)\left[\operatorname{esssup}_{y \in(0, x)} w(y)\right]^{r}\left(\int_{x}^{\infty} k(z, x) f(z) d z\right)^{r} d x\right)^{\frac{1}{r}} \leq \mathscr{A}_{0}\|f\|_{L_{v}^{p}}, \\
& \left(\int_{0}^{\infty} u(x)[\underset{y \in(0, x)}{\operatorname{ess} \sup }[w(y) k(x, y)]]^{r}\left(\int_{x}^{\infty} f\right)^{r} d x\right)^{\frac{1}{r}} \leq \mathscr{A}_{1}\|f\|_{L_{v}^{p}}
\end{aligned}
$$

for $q=\infty$ hold for all $f \in \mathfrak{M}^{+}$and the constant

$$
\mathscr{A}_{2}:= \begin{cases}\sup _{t \in(0, \infty)}\left(\int_{t}^{\infty} u\right)^{\frac{1}{r}}\left\|\mathscr{H}_{t}\right\|_{L_{v}^{p} \rightarrow L_{w}^{q},} & p \leq r, \\ \left(\int_{0}^{\infty} u(x)\left(\int_{x}^{\infty} u\right)^{\frac{s}{p}}\left\|\mathscr{H}_{\zeta^{-1}(x), \zeta(x)}\right\|_{L_{v}^{p} \rightarrow L_{w}^{q}}^{s} d x\right)^{\frac{1}{s}}, & r<p,\end{cases}
$$

is finite. Moreover, $C_{\mathscr{T}} \approx \mathscr{A}_{0}+\mathscr{A}_{1}+\mathscr{A}_{2}$.

Theorem 3.2. Let $1 \leq p<\infty, 0<r<\infty, 0<q \leq \infty, \frac{1}{s}:=\left(\frac{1}{r}-\frac{1}{p}\right)_{+}$. For validity of the inequality (1.4) it is necessary and sufficient that the inequalities

$$
\begin{aligned}
& \left(\int_{0}^{\infty} u(x)\left(\int_{\zeta^{-2}(x)}^{x} w\right)^{\frac{r}{q}}\left(\int_{0}^{\zeta^{-2}(x)} k\left(\zeta^{-2}(x), z\right) f(z) d z\right)^{r} d x\right)^{\frac{1}{r}} \leq \mathbf{A}_{0}\|f\|_{L_{v}^{p}} \\
& \left(\int_{0}^{\infty} u(x)\left(\int_{\zeta^{-2}(x)}^{x} w(y)\left[k\left(y, \zeta^{-2}(x)\right)\right]^{q} d y\right)^{\frac{r}{q}}\left(\int_{0}^{\zeta^{-2}(x)} f\right)^{r} d x\right)^{\frac{1}{r}} \leq \mathbf{A}_{1}\|f\|_{L_{v}^{p}}
\end{aligned}
$$

if $q<\infty$ or

$$
\begin{aligned}
& \left(\int_{0}^{\infty} u(x)\left[\operatorname{esssup}_{y \in\left(\zeta^{-2}(x), x\right)} w(y)\right]^{r}\left(\int_{0}^{\zeta^{-2}(x)} k\left(\zeta^{-2}(x), z\right) f(z) d z\right)^{r} d x\right)^{\frac{1}{r}} \leq \mathbf{A}_{0}\|f\|_{L_{v}^{p}}, \\
& \left(\int_{0}^{\infty} u(x)\left[\underset{y \in\left(\zeta^{-2}(x), x\right)}{\operatorname{ess} \sup }\left[w(y) k\left(y, \zeta^{-2}(x)\right)\right]\right]^{r}\left(\int_{0}^{\zeta^{-2}(x)} f\right)^{r} d x\right)^{\frac{1}{r}} \leq \mathbf{A}_{1}\|f\|_{L_{v}^{p}}
\end{aligned}
$$

for $q=\infty$ hold for all $f \in \mathfrak{M}^{+}$and the constant

$$
\mathbf{A}_{2}:= \begin{cases}\sup _{t \in(0, \infty)}\left(\int_{t}^{\infty} u\right)^{\frac{1}{r}}\left\|\mathscr{H}_{t}^{*}\right\|_{L_{v}^{p} \rightarrow L_{w}^{q},} & p \leq r, \\ \left(\int_{0}^{\infty} u(x)\left(\int_{x}^{\infty} u\right)^{\frac{s}{p}}\left\|\mathscr{H}_{\zeta^{-1}(x), \zeta(x)}^{*}\right\|_{L_{v}^{p} \rightarrow L_{w}^{q}}^{s} d x\right)^{\frac{1}{s}}, & r<p\end{cases}
$$

is finite. Moreover, $C_{\mathscr{S}} \approx \mathbf{A}_{0}+\mathbf{A}_{1}+\mathbf{A}_{2}$. 


\section{Operators $\mathrm{T}$ and $\mathrm{S}$}

Let the functions $\sigma$ and $\sigma^{-1}$ be the same as in the Section 2. For $0<c<d \leq \infty$ and $f \in \mathfrak{M}^{+}$ we put

$$
\begin{aligned}
& \left(\mathbf{H}_{c, d} f\right)(x):=\chi_{[c, d)}(x) \int_{\sigma^{-1}(c)}^{x} f(z) d z, \quad\left(\mathbf{H}_{c} f\right)(x):=\chi_{[c, \infty)}(x) \int_{0}^{x} f(z) d z \\
& \left(\mathbf{H}_{c, d}^{*} f\right)(x):=\chi_{[c, d)}(x) \int_{x}^{\sigma(d)} f(z) d z, \quad\left(\mathbf{H}_{c}^{*} f\right)(x):=\chi_{[c, \infty)}(x) \int_{x}^{\infty} f(z) d z .
\end{aligned}
$$

Theorem 4.1. Let $1 \leq p<\infty, 0<r<\infty, 0<q \leq \infty, \frac{1}{s}:=\left(\frac{1}{r}-\frac{1}{p}\right)_{+}$. For validity of the inequality (1.5) it is necessary and sufficient that

$$
B:=B_{0}+B_{1}+B_{2}<\infty
$$

where $B_{0}$ and $B_{1}$ are the least possible constants in the inequalities

$$
\left(\int_{0}^{\infty} u(x)\left(\int_{x}^{\infty} k(y, x) w(y) d y\right)^{\frac{r}{q}}\left(\int_{0}^{x} f\right)^{r} d x\right)^{\frac{1}{r}} \leq B_{0}\|f\|_{L_{v}^{p}}
$$

and

$$
\left(\int_{0}^{\infty} u(x)\left[k\left(\sigma^{2}(x), x\right)\right]^{\frac{r}{q}}\left(\int_{\sigma^{2}(x)}^{\infty} w(y)\left(\int_{0}^{y} f\right)^{q} d y\right)^{\frac{r}{q}} d x\right)^{\frac{1}{r}} \leq B_{1}\|f\|_{L_{v}^{p}}
$$

if $q<\infty$ or

$$
\begin{aligned}
& \left(\int_{0}^{\infty} u(x)\left[\operatorname{esssp}_{y \geq x} k(y, x) w(y)\right]^{r}\left(\int_{0}^{x} f\right)^{r} d x\right)^{\frac{1}{r}} \leq B_{0}\|f\|_{L_{v}^{p}}, \\
& \left(\int_{0}^{\infty} u(x)\left[k\left(\sigma^{2}(x), x\right)\right]^{r}\left(\underset{y \geq \sigma^{2}(x)}{\operatorname{ess} \sup } w(y) \int_{0}^{y} f\right)^{r} d x\right)^{\frac{1}{r}} \leq B_{1}\|f\|_{L_{v}^{p}},
\end{aligned}
$$

for $q=\infty$ and $B_{2}$ is defined by

$$
B_{2}:= \begin{cases}\sup _{t>0}\left(\int_{0}^{t} u\right)^{\frac{1}{r}}\left\|\mathbf{H}_{t}\right\|_{L_{v}^{p} \rightarrow L_{w(\cdot) k(\cdot, t)}^{q},}, & p \leq r, \\ \left(\int_{0}^{\infty} u(x)\left(\int_{0}^{x} u\right)^{\frac{s}{p}}\left\|\mathbf{H}_{\sigma^{-1}(x), \sigma^{2}(x)}\right\|_{\left.L_{v}^{p} \rightarrow L_{w(\cdot) k\left(\cdot, \sigma^{-1}(x)\right)}^{s} d x\right)^{\frac{1}{s}},}, \quad r<p .\right.\end{cases}
$$

Moreover, $C_{\mathbf{T}} \approx B$.

Proof. Let the sequence $\left\{a_{n}\right\}$ be the same as in the proof of Theorem 2.1 and $q<\infty$.

Sufficiency. We write

$$
\begin{aligned}
J & :=\int_{0}^{\infty}[T f]^{r} u=\sum_{n \leq N} \int_{a_{n}}^{a_{n+1}}[T f]^{r} u \\
& \approx \sum_{n \leq N} 2^{n}\left(\int_{a_{n}}^{\infty} k\left(y, a_{n}\right) w(y)\left(\int_{0}^{y} f\right)^{q} d y\right)^{\frac{r}{q}} \approx J_{1}+J_{2},
\end{aligned}
$$


where

$$
\begin{aligned}
J_{1} & :=\sum_{n \leq N} 2^{n}\left(\int_{a_{n}}^{a_{n+2}} k\left(y, a_{n}\right) w(y)\left(\int_{0}^{y} f\right)^{q} d y\right)^{\frac{r}{q}}, \\
J_{2} & :=\sum_{n \leq N} 2^{n}\left(\int_{a_{n+2}}^{\infty} k\left(y, a_{n}\right) w(y)\left(\int_{0}^{y} f\right)^{q} d y\right)^{\frac{r}{q}} .
\end{aligned}
$$

Estimate of $J_{1}$. We have

$$
\begin{aligned}
J_{1} & \approx \sum_{n \leq N} 2^{n}\left(\int_{a_{n}}^{a_{n+2}} k\left(y, a_{n}\right) w(y)\left(\int_{a_{n-1}}^{y} f\right)^{q} d y\right)^{\frac{r}{q}} \\
& +\sum_{n \leq N} 2^{n}\left(\int_{a_{n}}^{a_{n+2}} k\left(y, a_{n}\right) w(y) d y\right)^{\frac{r}{q}}\left(\int_{0}^{a_{n-1}} f\right)^{r}=J_{1,1}+J_{1,2} .
\end{aligned}
$$

For $J_{1,2}$ we write

$$
\begin{aligned}
J_{1,2} & \approx \sum_{n \leq N} \int_{a_{n-1}}^{a_{n}} u(x) d x\left(\int_{a_{n}}^{a_{n+2}} k\left(y, a_{n}\right) w(y) d y\right)^{\frac{r}{q}}\left(\int_{0}^{a_{n-1}} f\right)^{r} \\
& \lesssim \int_{0}^{\infty} u(x)\left(\int_{x}^{\infty} k(y, x) w(y) d y\right)^{\frac{r}{q}}\left(\int_{0}^{x} f\right)^{r} d x \leq B_{0}^{r}\left(\int_{0}^{\infty} f^{p} v\right)^{\frac{r}{p}} .
\end{aligned}
$$

For $J_{1,1}$ we write

$$
\begin{aligned}
J_{1,1} & \approx \sum_{n \leq N} 2^{n}\left(\int_{a_{n}}^{a_{n+2}} k\left(y, a_{n}\right) w(y)\left(\mathbf{H}_{a_{n}, a_{n+2}} f(y)\right)^{q} d y\right)^{\frac{r}{q}} \\
& \lesssim \sum_{n \leq N} 2^{n}\left\|\mathbf{H}_{a_{n}, a_{n+2}}\right\|_{L_{v}^{p} \rightarrow L_{w(\cdot) k\left(\cdot, a_{n}\right)}^{q}}^{r}\left(\int_{a_{n-1}}^{a_{n+2}} f^{p} v\right)^{\frac{r}{p}} .
\end{aligned}
$$

If $p \leq r$, by Jensen's inequality we get

$$
J_{1,1} \lesssim B_{2}^{r}\|f\|_{L_{v}^{p}}^{r}
$$

If $r<p$, by Hölder's inequality,

$$
\begin{aligned}
J_{1,1} & \lesssim\left(\sum_{n \leq N}\left(2^{n}\right)^{\frac{s}{r}}\left\|\mathbf{H}_{a_{n}, a_{n+2}}\right\|_{L_{v}^{p} \rightarrow L_{w(\cdot) k\left(\cdot, a_{n}\right)}^{q}}^{s}\right)^{\frac{r}{s}}\|f\|_{L_{v}^{p}}^{r} \\
& \lesssim\left(\sum_{n \leq N} \int_{a_{n}}^{a_{n+1}} u\left(\int_{0}^{a_{n}} u\right)^{\frac{s}{p}}\left\|\mathbf{H}_{\sigma^{-1}\left(a_{n+1}\right), \sigma^{2}\left(a_{n}\right)}\right\|_{L_{v}^{p} \rightarrow L_{w(\cdot) k\left(\cdot, \sigma^{-1}\left(a_{n+1}\right)\right)}^{s}}\right)^{\frac{r}{s}}\|f\|_{L_{v}^{p}}^{r} \\
& \lesssim B_{2}^{r}\|f\|_{L_{v}^{p}}^{r} .
\end{aligned}
$$

Thus,

$$
J_{1} \lesssim\left(B_{0}+B_{2}\right)^{r}\|f\|_{L_{v}^{p}}^{r}
$$


Estimate of $J_{2}$. Denote $h(y):=w(y)\left(\int_{0}^{y} f\right)^{q}$ and using (1.9) we obtain

$$
\begin{aligned}
& \int_{a_{n+2}}^{\infty} k\left(y, a_{n}\right) h(y) d y=\sum_{i \geq n} \int_{a_{i+2}}^{a_{i+3}} k\left(y, a_{n}\right) h(y) d y \\
& \quad \approx \sum_{i \geq n} \int_{a_{i+2}}^{a_{i+3}} k\left(y, a_{i+1}\right) h(y) d y+\sum_{i \geq n} \int_{a_{i+2}}^{a_{i+3}} k\left(a_{i+1}, a_{n}\right) h(y) d y \\
& \quad \lesssim \sum_{i \geq n} \int_{a_{i+1}}^{a_{i+3}} k\left(y, a_{i+1}\right) h(y) d y+\sum_{i \geq n} \int_{a_{i+2}}^{a_{i+3}} k\left(a_{i+1}, a_{n}\right) h(y) d y=: I_{1, n}+I_{2, n}
\end{aligned}
$$

Similar to the proof of (4.7) we find

$$
\sum_{n \leq N} 2^{n} I_{1, n}^{\frac{r}{q}} \approx J_{1} \lesssim\left(B_{0}+B_{2}\right)^{r}\|f\|_{L_{v}^{p}}^{r} .
$$

By [15], Lemma 3.1 there is $\alpha \in(0,1)$ such that

$$
k\left(a_{i+1}, a_{n}\right) \lesssim\left(\sum_{j=n}^{i}\left[k\left(a_{j+1}, a_{j}\right)\right]^{\alpha}\right)^{\frac{1}{\alpha}}, i \geq n .
$$

By Minkowskii's inequality

$$
\begin{aligned}
I_{2, n} & \lesssim \sum_{i \geq n}\left(\sum_{j=n}^{i}\left[k\left(a_{j+1}, a_{j}\right)\right]^{\alpha}\right)^{\frac{1}{\alpha}} \int_{a_{i+2}}^{a_{i+3}} h(y) d y \\
& \leq\left(\sum_{j \geq n}\left[k\left(a_{j+1}, a_{j}\right)\right]^{\alpha}\left(\int_{a_{j+2}}^{\infty} h\right)^{\alpha}\right)^{\frac{1}{\alpha}} .
\end{aligned}
$$

Hence,

$$
\begin{aligned}
\sum_{n \leq N} 2^{n} I_{2, n}^{\frac{r}{q}} & \leq \sum_{n \leq N} 2^{n}\left(\sum_{j \geq n}\left[k\left(a_{j+1}, a_{j}\right)\right]^{\alpha}\left(\int_{a_{j+2}}^{\infty} h\right)^{\alpha}\right)^{\frac{r}{q \alpha}} \\
& \approx \sum_{n \leq N} 2^{n} k\left(a_{n+1}, a_{n}\right)^{\frac{r}{q}}\left(\int_{a_{n+2}}^{\infty} h\right)^{\frac{r}{q}} \\
& \approx \sum_{n \leq N}\left[\int_{a_{n-1}}^{a_{n}} u\right] k\left(\sigma^{2}\left(a_{n-1}\right), a_{n}\right)^{\frac{r}{q}}\left(\int_{\sigma^{2}\left(a_{n}\right)}^{\infty} h\right)^{\frac{r}{q}} \\
& \lesssim \int_{0}^{\infty} u(x) k\left(\sigma^{2}(x), x\right)^{\frac{r}{q}}\left(\int_{\sigma^{2}(x)}^{\infty} w(y)\left(\int_{0}^{y} f\right)^{q} d y\right)^{\frac{r}{q}} d x \leq B_{1}^{r}\|f\|_{L_{v}^{p}}^{r} .
\end{aligned}
$$

In case of $q=\infty$ we write

$$
\begin{aligned}
& \operatorname{ess} \sup _{y \in\left[a_{n+2}, \infty\right)} k\left(y, a_{n}\right) h(y)=\sup _{i \geq n} \operatorname{esssup}_{y \in\left[a_{i+2}, a_{i+3}\right)} k\left(y, a_{n}\right) h(y) \\
& \lesssim \sup _{i \geq n} \operatorname{ess}_{y \in\left[a_{i+1}, a_{i+3}\right)} k\left(y, a_{i+1}\right) h(y)+\sup _{i \geq n} k\left(a_{i+1}, a_{n}\right) \underset{y \in\left[a_{i+2}, a_{i+3}\right)}{\operatorname{ess} \sup _{i+n}} h(y)=: I_{1, n}+I_{2, n} .
\end{aligned}
$$


The estimate (4.8) follows by the same way. Also we have

$$
\begin{aligned}
I_{2, n} & \lesssim\left(\sup _{i \geq n} \sum_{j=n}^{i}\left[k\left(a_{j+1}, a_{j}\right)\right]^{\alpha}\left(\operatorname{esssup}_{y \in\left[a_{i+2}, a_{i+3}\right)} h(y)\right)^{\alpha}\right)^{\frac{1}{\alpha}} \\
& \leq\left(\sum_{j \geq n}\left[k\left(a_{j+1}, a_{j}\right)\right]^{\alpha} \sup _{i \geq n}\left(\operatorname{ess~sup}_{y \in\left[a_{i+2}, a_{i+3}\right)} h(y)\right)^{\alpha} \chi_{[n, i]}(j)\right)^{\frac{1}{\alpha}} \\
& =\left(\sum_{j \geq n}\left[k\left(a_{j+1}, a_{j}\right)\right]^{\alpha}\left(\operatorname{ess~sup~}_{y \in\left[a_{j+2}, \infty\right)}^{\operatorname{esc}} h(y)\right)^{\alpha}\right)^{\frac{1}{\alpha}}
\end{aligned}
$$

and the inequality $\sum_{n \leq N} 2^{n} I_{2, n}^{\frac{r}{q}} \lesssim B_{1}^{r}\|f\|_{L_{v}^{p}}^{r}$ follows for this case too. Thus,

$$
J_{2} \lesssim\left(B_{0}+B_{1}+B_{2}\right)^{r}\|f\|_{L_{v}^{p}}^{r}
$$

and the upper bound $C_{\mathbf{T}} \lesssim B_{0}+B_{1}+B_{2}$ is proved.

Necessity. Suppose the inequality (1.5) hold, that is

$$
\left(\int_{0}^{\infty}\left(\int_{x}^{\infty} k(y, x) w(y)\left(\int_{0}^{y} f\right)^{q} d y\right)^{\frac{r}{q}} u\right)^{\frac{1}{r}} \leq C_{\mathbf{T}}\left(\int_{0}^{\infty} f^{p} v\right)^{\frac{1}{p}}
$$

for all $f \in \mathfrak{M}^{+}$. Narrowing the integration $(0, y) \rightarrow(0, x)$ on the left-hand side, we see, that $C_{\mathbf{T}} \geq B_{0}$. Analogously, if $(x, \infty) \rightarrow\left(\sigma^{2}(x), \infty\right), k(y, x) \gtrsim k\left(\sigma^{2}(x), x\right)$, then $C_{\mathbf{T}} \geq B_{1}$. If $(0, \infty) \rightarrow(0, t),(x, \infty) \rightarrow(t, \infty), k(y, x) \gtrsim k(y, t)$, then

$$
C_{\mathbf{T}} \geq\left(\int_{0}^{t} u\right)^{\frac{1}{r}}\left\|\mathbf{H}_{t}\right\|_{L_{v}^{p} \rightarrow L_{w(\cdot) k(\cdot, t)}^{q}}
$$

for all $t>0$. Consequently, $C_{\mathbf{T}} \gtrsim B_{2}$ in case of $p \leq r$.

In the case $r<p$ we write

$$
\begin{aligned}
B_{2}^{s} & =\int_{0}^{\infty} u(x)\left(\int_{0}^{x} u\right)^{\frac{s}{p}}\left\|\mathbf{H}_{\sigma^{-1}(x), \sigma^{2}(x)}\right\|_{L_{v}^{p} \rightarrow L_{w(\cdot) k\left(\cdot, \sigma^{-1}(x)\right)}^{s}}^{q} d x \\
& =\sum_{n \leq N} \int_{a_{n}}^{a_{n+1}} u(x)\left(\int_{0}^{x} u\right)^{\frac{s}{p}}\left\|\mathbf{H}_{\sigma^{-1}(x), \sigma^{2}(x)}\right\|_{L_{v}^{p} \rightarrow L_{w(\cdot) k\left(\cdot, \sigma^{-1}(x)\right)}^{q}}^{q} d x \\
& \leq \sum_{n \leq N}\left(\int_{a_{n}}^{a_{n+1}} u\right)\left(\int_{0}^{a_{n+1}} u\right)^{\frac{s}{p}}\left\|\mathbf{H}_{\sigma^{-1}\left(a_{n}\right), \sigma^{2}\left(a_{n+1}\right)}\right\|_{L_{v}^{p} \rightarrow L_{w(\cdot) k\left(\cdot, \sigma^{-1}\left(a_{n}\right)\right)}^{q}}^{q}=: \bar{B}_{2}^{s .} \\
& \approx \sum_{n \leq N}\left(2^{n}\right)^{\frac{s}{r}}\left\|\mathbf{H}_{a_{n-1}, a_{n+3}}\right\|_{L_{v}^{p} \rightarrow L_{w(\cdot) k\left(\cdot, a_{n-1}\right)}^{q}}^{s}
\end{aligned}
$$

Let $\theta \in(0,1)$ be arbitrary. Then for all $n \leq N$ there is $f_{n} \in \mathfrak{M}^{+}$such that $\operatorname{supp} f_{n} \subset$ $\left[a_{n-2}, a_{n+3}\right],\left\|f_{n}\right\|_{L_{v}^{p}}=1$ and

$$
\left\|\mathbf{H}_{a_{n-1}, a_{n+3}} f_{n}\right\|_{L_{w(\cdot) k\left(\cdot, a_{n-1}\right)}^{q}} \geq \theta\left\|\mathbf{H}_{a_{n-1}, a_{n+3}}\right\|_{L_{v}^{p} \rightarrow L_{w(\cdot) k\left(\cdot, a_{n-1}\right)}^{q}} .
$$


Put

$$
g_{n}:=\left(2^{n}\right)^{\frac{s}{p r}}\left\|\mathbf{H}_{a_{n-1}, a_{n+3}}\right\|_{L_{v}^{p} \rightarrow L_{w(\cdot) k\left(\cdot, a_{n-1}\right)}^{\frac{s}{p}}} f_{n}, \quad g:=\sum_{n \leq N} g_{n} .
$$

We have

$$
\begin{aligned}
\|g\|_{L_{v}^{p}}^{p} & =\sum_{j \leq N} \int_{a_{j}}^{a_{j+1}}\left(\sum_{n \leq N} g_{n}(x)\right)^{p} v(x) d x \\
& =\sum_{j \leq N} \int_{a_{j}}^{a_{j+1}}\left(\sum_{n=j-2}^{j+2} g_{n}(x)\right)^{p} v(x) d x \\
& \lesssim \sum_{j \leq N} \int_{a_{j-2}}^{a_{j+3}} g_{j}(x)^{p} v(x) d x \\
& =\sum_{j \leq N}\left(2^{j}\right)^{\frac{s}{r}}\left\|\mathbf{H}_{a_{j-1}, a_{j+3}}\right\|_{L_{v}^{p} \rightarrow L_{w(\cdot) k\left(\cdot, a_{j-1}\right)}^{q}}^{q}=\bar{B}_{2}^{s} .
\end{aligned}
$$

Finally, applying (1.1)

$$
\begin{aligned}
C_{\mathbf{T}}^{r} \mathscr{A}^{\frac{s r}{p}} & \gtrsim C_{\mathbf{T}}^{r}\|g\|_{L_{v}^{p}}^{r} \geq \int_{0}^{\infty}[T g]^{r} u \geq \sum_{n \leq N} \int_{a_{n-2}}^{a_{n-1}}[\mathbf{T} g]^{r} u \\
& \geq \sum_{n \leq N}\left(\int_{a_{n-2}}^{a_{n-1}} u\right)\left\|\mathbf{H}_{a_{n-1}, a_{n+3}} g\right\|_{L_{w(\cdot) k\left(\cdot, a_{n-1}\right)}^{r}}^{r} \gtrsim \sum_{n \leq N} 2^{n}\left\|\mathbf{H}_{a_{n-1}, a_{n+3}} g_{n}\right\|_{L_{w(\cdot) k\left(\cdot, a_{n-1}\right)}^{r}}^{r} \geq \theta^{r} \bar{B}_{2}^{s} . \\
& =\sum_{n \leq N}\left(2^{n}\right)^{\frac{s}{r}}\left\|\mathbf{H}_{a_{n-1}, a_{n+3}}\right\|_{L_{v}^{p} \rightarrow L_{w(\cdot) k\left(\cdot, a_{n-1}\right)}^{q}}^{\frac{s r}{p}}\left\|\mathbf{H}_{a_{n-1}, a_{n+3}} f_{n}\right\|_{L_{w(\cdot) k\left(\cdot, a_{n-1}\right)}^{r}}^{r},
\end{aligned}
$$

Thus, $C_{\mathbf{T}} \gtrsim \theta \bar{B}_{2}$. Hence, $C_{\mathbf{T}} \gtrsim \theta B_{2}$ and the required lower bound $C_{\mathbf{T}} \gtrsim B_{0}+B_{1}+B_{2}$ follows.

Remark 4.1. Similar to $(2.8)$ and $(2.9)$ we have

$$
\begin{gathered}
C_{\mathbf{T}}=\left\|\mathbf{T}\left(\frac{1}{v}\right)\right\|_{L_{u}^{r}}, p=\infty, \\
C_{\mathbf{T}} \approx \sup _{t \geq 0} U(t)\left\|\mathbf{H}_{t}\right\|_{L_{v}^{p} \rightarrow L_{w(\cdot) k(\cdot, t)}^{q}}, r=\infty .
\end{gathered}
$$

Theorem 4.2. Let $1 \leq p<\infty, 0<r<\infty, 0<q \leq \infty, \frac{1}{s}:=\left(\frac{1}{r}-\frac{1}{p}\right)_{+}$. For validity of the inequality (1.7) it is necessary and sufficient that

$$
\mathbb{B}:=\mathbb{B}_{0}+\mathbb{B}_{1}+\mathbb{B}_{2}<\infty
$$

where $\mathbb{B}_{0}$ and $\mathbb{B}_{1}$ are the least possible constants in the inequalities

$$
\left(\int_{0}^{\infty} u(x)\left(\int_{x}^{\sigma^{3}(x)} k(y, x) w(y) d y\right)^{\frac{r}{q}}\left(\int_{\sigma^{3}(x)}^{\infty} f\right)^{r} d x\right)^{\frac{1}{r}} \leq \mathbb{B}_{0}\|f\|_{L_{v}^{p}}
$$


and

$$
\left(\int_{0}^{\infty} u(x) k\left(\sigma^{2}(x), x\right)^{\frac{r}{q}}\left(\int_{\sigma^{2}(x)}^{\infty} w(y)\left(\int_{y}^{\infty} f\right)^{q} d y\right)^{\frac{r}{q}} d x\right)^{\frac{1}{r}} \leq \mathbb{B}_{1}\|f\|_{L_{v}^{p}}
$$

when $q<\infty$ and

$$
\left(\int_{0}^{\infty} u(x)\left[\operatorname{esssup}_{x \leq y \leq \sigma^{3}(x)} k(y, x) w(y)\right]^{r}\left(\int_{\sigma^{3}(x)}^{\infty} f\right)^{r} d x\right)^{\frac{1}{r}} \leq \mathbb{B}_{0}\|f\|_{L_{v}^{p}},
$$

and

$$
\left(\int_{0}^{\infty} u(x)\left[k\left(\sigma^{2}(x), x\right)\right]^{r}\left(\underset{y \geq \sigma^{2}(x)}{\operatorname{essup} w(y)} \int_{y}^{\infty} f\right)^{r} d x\right)^{\frac{1}{r}} \leq \mathbb{B}_{1}\|f\|_{L_{v}^{p}},
$$

if $q=\infty$. The constant $\mathbb{B}_{2}$ is given by

$$
\mathbb{B}_{2}:= \begin{cases}\sup _{t>0}\left(\int_{0}^{t} u\right)^{\frac{1}{r}}\left\|\mathbf{H}_{t}^{*}\right\|_{L_{v}^{p} \rightarrow L_{w(\cdot) k(\cdot, t)}^{q},}, & p \leq r, \\ \left(\int_{0}^{\infty} u(x)\left(\int_{0}^{x} u\right)^{\frac{s}{p}}\left\|\mathbf{H}_{\sigma^{-1}(x), \sigma^{2}(x)}^{*}\right\|_{\left.L_{v}^{p} \rightarrow L_{w(\cdot) k\left(\cdot, \sigma^{-1}(x)\right)}^{q} d x\right)^{\frac{1}{s}},}, \quad r<p .\right.\end{cases}
$$

Moreover, $C_{\mathbf{S}} \approx \mathbb{B}$.

Proof. Let the sequence $\left\{a_{n}\right\}$ be the same as in the proof of Theorem 4.1 and $q<\infty$.

Sufficiency. We write

$$
\begin{aligned}
J & :=\int_{0}^{\infty}[\mathbf{S} f]^{r} u=\sum_{n \leq N} \int_{a_{n}}^{a_{n+1}}[\mathbf{S} f]^{r} u \\
& \approx \sum_{n \leq N} 2^{n}\left(\int_{a_{n}}^{\infty} k\left(y, a_{n}\right) w(y)\left(\int_{y}^{\infty} f\right)^{q} d y\right)^{\frac{r}{q}} \approx J_{1}+J_{2},
\end{aligned}
$$

where

$$
\begin{aligned}
& J_{1}:=\sum_{n \leq N} 2^{n}\left(\int_{a_{n}}^{a_{n+2}} k\left(y, a_{n}\right) w(y)\left(\int_{y}^{\infty} f\right)^{q} d y\right)^{\frac{r}{q}}, \\
& J_{2}:=\sum_{n \leq N} 2^{n}\left(\int_{a_{n+2}}^{\infty} k\left(y, a_{n}\right) w(y)\left(\int_{y}^{\infty} f\right)^{q} d y\right)^{\frac{r}{q}} .
\end{aligned}
$$

Estimate of $J_{1}$. We have

$$
\begin{aligned}
J_{1} & \approx \sum_{n \leq N} 2^{n}\left(\int_{a_{n}}^{a_{n+2}} k\left(y, a_{n}\right) w(y)\left(\int_{y}^{a_{n+3}} f\right)^{q} d y\right)^{\frac{r}{q}} \\
& +\sum_{n \leq N} 2^{n}\left(\int_{a_{n}}^{a_{n+2}} k\left(y, a_{n}\right) w(y) d y\right)^{\frac{r}{q}}\left(\int_{a_{n+3}}^{\infty} f\right)^{r}=J_{1,1}+J_{1,2} .
\end{aligned}
$$


For $J_{1,2}$ we write

$$
\begin{aligned}
J_{1,2} & \approx \sum_{n \leq N} \int_{a_{n-1}}^{a_{n}} u(x) d x\left(\int_{a_{n}}^{a_{n+2}} k\left(y, a_{n}\right) w(y) d y\right)^{\frac{r}{q}}\left(\int_{a_{n+3}}^{\infty} f\right)^{r} \\
& \lesssim \int_{0}^{\infty} u(x)\left(\int_{x}^{\sigma^{3}(x)} k(y, x) w(y) d y\right)^{\frac{r}{q}}\left(\int_{\sigma^{3}(x)}^{\infty} f\right)^{r} d x \leq \mathbb{B}_{0}^{r}\left(\int_{0}^{\infty} f^{p} v\right)^{\frac{r}{p}} .
\end{aligned}
$$

For $J_{1,1}$ we write

$$
\begin{aligned}
J_{1,1} & \approx \sum_{n \leq N} 2^{n}\left(\int_{a_{n}}^{a_{n+2}} k\left(y, a_{n}\right) w(y)\left(\mathbf{H}_{a_{n}, a_{n+2}}^{*} f(y)\right)^{q} d y\right)^{\frac{r}{q}} \\
& \lesssim \sum_{n \leq N} 2^{n}\left\|\mathbf{H}_{a_{n}, a_{n+2}}^{*}\right\|_{L_{v}^{p} \rightarrow L_{w(\cdot) k\left(\cdot, a_{n}\right)}^{q}}\left(\int_{a_{n}}^{a_{n+3}} f^{p} v\right)^{\frac{r}{p}} .
\end{aligned}
$$

If $p \leq r$ then, by Jensen's inequality, we get

$$
J_{1,1} \lesssim \mathbb{B}_{2}^{r}\|f\|_{L_{v}^{p}}^{r}
$$

If $r<p$ then, by Hölder's inequality,

$$
\begin{aligned}
J_{1,1} & \lesssim\left(\sum_{n \leq N}\left(2^{n}\right)^{\frac{s}{r}}\left\|\mathbf{H}_{a_{n}, a_{n+2}}^{*}\right\|_{L_{v}^{p} \rightarrow L_{w(\cdot) k\left(\cdot, a_{n}\right)}^{q}}^{s}\right)^{\frac{r}{s}}\|f\|_{L_{v}^{p}}^{r} \\
& \lesssim\left(\sum_{n \leq N} \int_{a_{n}}^{a_{n+1}} u\left(\int_{0}^{a_{n}} u\right)^{\frac{s}{p}}\left\|\mathbf{H}_{\sigma^{-1}\left(a_{n+1}\right), \sigma^{2}\left(a_{n}\right)}^{*}\right\|_{L_{v}^{p} \rightarrow L_{w(\cdot) k\left(\cdot, \sigma^{-1}\left(a_{n+1}\right)\right)}^{s}}\right)^{\frac{r}{s}}\|f\|_{L_{v}^{p}}^{r} \\
& \lesssim \mathbb{B}_{2}^{r}\|f\|_{L_{v}^{p}}^{r}
\end{aligned}
$$

Thus

$$
J_{1} \lesssim\left(\mathbb{B}_{0}+\mathbb{B}_{2}\right)^{r}\|f\|_{L_{v}^{p}}^{r}
$$

Estimate of $J_{2}$. Denote $h(y):=w(y)\left(\int_{y}^{\infty} f\right)^{q}$ and arguing similar to the proof of Theorem 4.1 we obtain

$$
J_{2} \lesssim\left(\mathbb{B}_{0}+\mathbb{B}_{1}+\mathbb{B}_{2}\right)^{r}\|f\|_{L_{v}^{p}}^{r}
$$

Necessity. Suppose that the inequality (1.3) holds, that is

$$
\left(\int_{0}^{\infty}\left(\int_{x}^{\infty} k(y, x) w(y)\left(\int_{y}^{\infty} f\right)^{q} d y\right)^{\frac{r}{q}} u\right)^{\frac{1}{r}} \leq C_{S}\left(\int_{0}^{\infty} f^{p} v\right)^{\frac{1}{p}}
$$

for all $f \in \mathfrak{M}^{+}$. Narrowing the integration $(x, \infty) \rightarrow\left(x, \sigma^{3}(x)\right)$ and $(y, \infty) \rightarrow\left(\sigma^{3}(x), \infty\right)$ on the left-hand side, we see, that $C_{\mathbf{S}} \geq \mathbb{B}_{0}$. Analogously, if $(x, \infty) \rightarrow\left(\sigma^{2}(x), \infty\right), k(y, x) \gtrsim$ $k\left(\sigma^{2}(x), x\right)$, then $C_{\mathbf{S}} \geq \mathbb{B}_{1}$. The proof of $C_{\mathbf{S}} \gtrsim \mathbb{B}_{2}$ is similar to the proof of $C_{\mathbf{T}} \gtrsim B_{2}$.

Remark 4.2. Similar to $(2.15)$ and (2.16) the equalities

$$
C_{\mathbf{S}}=\left\|\mathbf{S}\left(\frac{1}{v}\right)\right\|_{L_{u}^{r}}, p=\infty
$$


and

$$
C_{\mathbf{S}} \approx \sup _{t \geq 0} U(t)\left\|\mathbf{H}_{t}^{*}\right\|_{L_{v}^{p} \rightarrow L_{w(\cdot) k(\cdot, t)}^{q}}, r=\infty
$$

hold true.

\section{Operators $\mathfrak{T}$ and $\mathfrak{S}$}

Let the functions $\zeta, \zeta^{-1}:[0, \infty) \rightarrow[0, \infty)$ be the same as in the Section 3. For $0 \leq c<d<\infty$ and $f \in \mathfrak{M}^{+}$we define operators

$$
\begin{aligned}
\left(\mathfrak{H}_{c, d} f\right)(x) & :=\chi_{(c, d]}(x) \int_{x}^{\zeta(d)} f(z) d z, \\
\left(\mathfrak{H}_{d} f\right)(x) & :=\chi_{(0, d]}(x) \int_{x}^{\infty} f(z) d z, \\
\left(\mathfrak{H}_{c, d}^{*} f\right)(x) & :=\chi_{(c, d]}(x) \int_{\zeta^{-1}(c)}^{x} f(z) d z, \\
\left(\mathfrak{H}_{d}^{*} f\right)(x) & :=\chi_{(0, d]}(x) \int_{0}^{x} f(z) d z .
\end{aligned}
$$

The following theorems are true.

Theorem 5.1. Let $1 \leq p<\infty, 0<r<\infty, 0<q \leq \infty, \frac{1}{s}:=\left(\frac{1}{r}-\frac{1}{p}\right)_{+}$. For validity of the inequality (1.6) it is necessary and sufficient that the inequalities

$$
\begin{aligned}
& \left(\int_{0}^{\infty} u(x)\left(\int_{0}^{x} k(x, y) w(y) d y\right)^{\frac{r}{q}}\left(\int_{x}^{\infty} f\right)^{r} d x\right)^{\frac{1}{r}} \leq \mathscr{B}_{0}\|f\|_{L_{v}^{p}} \\
& \left(\int_{0}^{\infty} u(x)\left[k\left(x, \zeta^{-2}(x)\right)\right]^{\frac{r}{q}}\left(\int_{0}^{\zeta^{-2}(x)} w(y)\left(\int_{y}^{\infty} f\right)^{q} d y\right)^{\frac{r}{q}} d x\right)^{\frac{1}{r}} \leq \mathscr{B}_{1}\|f\|_{L_{v}^{p}},
\end{aligned}
$$

if $q<\infty$ or

$$
\begin{aligned}
& \left(\int_{0}^{\infty} u(x)\left[\operatorname{esssup}_{y \in(0, x)} k(x, y) w(y)\right]^{r}\left(\int_{x}^{\infty} f\right)^{r} d x\right)^{\frac{1}{r}} \leq \mathscr{B}_{0}\|f\|_{L_{v}^{p}}, \\
& \left(\int_{0}^{\infty} u(x)\left[k\left(x, \zeta^{-2}(x)\right)\right]^{r}\left(\operatorname{essup}_{y \in\left(0, \zeta^{-2}(x)\right)} w(y) \int_{y}^{\infty} f\right)^{r} d x\right)^{\frac{1}{r}} \leq \mathscr{B}_{1}\|f\|_{L_{v}^{p}}
\end{aligned}
$$

for $q=\infty$ hold for all $f \in \mathfrak{M}^{+}$and the constant

$$
\mathscr{B}_{2}:= \begin{cases}\sup _{t \in(0, \infty)}\left(\int_{t}^{\infty} u\right)^{\frac{1}{r}}\left\|\mathfrak{H}_{t}\right\|_{L_{v}^{p} \rightarrow L_{w(\cdot) k(t, \cdot)}^{q},} & p \leq r, \\ \left(\int_{0}^{\infty} u(x)\left(\int_{x}^{\infty} u\right)^{\frac{s}{p}}\left\|\mathfrak{H}_{\zeta^{-1}(x), \zeta^{2}(x)}\right\|_{\left.L_{v}^{p} \rightarrow L_{w(\cdot) k\left(\zeta^{2}(x), \cdot\right)}^{q} d x\right)^{\frac{1}{s}},},\right. & r<p,\end{cases}
$$

is finite. Moreover, $C_{\mathfrak{T}} \approx \mathscr{B}_{0}+\mathscr{B}_{1}+\mathscr{B}_{2}$. 
Theorem 5.2. Let $1 \leq p<\infty, 0<r<\infty, 0<q \leq \infty, \frac{1}{s}:=\left(\frac{1}{r}-\frac{1}{p}\right)_{+}$. For validity of the inequality (1.8) it is necessary and sufficient that the inequalities

$$
\begin{aligned}
& \left(\int_{0}^{\infty} u(x)\left(\int_{\zeta^{-3}(x)}^{x} k(x, y) w(y) d y\right)^{\frac{r}{q}}\left(\int_{0}^{\zeta^{-3}(x)} f\right)^{r} d x\right)^{\frac{1}{r}} \leq \mathbf{B}_{0}\|f\|_{L_{v}^{p}}, \\
& \left(\int_{0}^{\infty} u(x)\left[k\left(x, \zeta^{-2}(x)\right)\right]^{\frac{r}{q}}\left(\int_{0}^{\zeta^{-2}(x)} w(y)\left(\int_{0}^{y} f\right)^{q} d y\right)^{\frac{r}{q}} d x\right)^{\frac{1}{r}} \leq \mathbf{B}_{1}\|f\|_{L_{v}^{p}},
\end{aligned}
$$

if $q<\infty$ or

$$
\begin{aligned}
& \left(\int_{0}^{\infty} u(x)\left[\operatorname{esssup}_{y \in\left(\zeta^{-3}(x), x\right)} k(x, y) w(y)\right]^{r}\left(\int_{0}^{\zeta^{-3}(x)} f\right)^{r} d x\right)^{\frac{1}{r}} \leq \mathbf{B}_{0}\|f\|_{L_{v}^{p}}, \\
& \left(\int_{0}^{\infty} u(x)\left[k\left(x, \zeta^{-2}(x)\right)\right]^{r}\left(\operatorname{esssup}_{y \in\left(0, \zeta^{-2}(x)\right)} w(y) \int_{0}^{y} f\right)^{r} d x\right)^{\frac{1}{r}} \leq \mathbf{B}_{1}\|f\|_{L_{v}^{p}}
\end{aligned}
$$

for $q=\infty$ hold for all $f \in \mathfrak{M}^{+}$and the constant

$$
\mathbf{B}_{2}:= \begin{cases}\sup _{t \in(0, \infty)}\left(\int_{t}^{\infty} u\right)^{\frac{1}{r}}\left\|\mathfrak{H}_{t}^{*}\right\|_{L_{v}^{p} \rightarrow L_{w(\cdot) k(t, \cdot)}^{q},} & p \leq r, \\ \left(\int_{0}^{\infty} u(x)\left(\int_{x}^{\infty} u\right)^{\frac{s}{p}}\left\|\mathfrak{H}_{\zeta^{-1}(x), \zeta^{2}(x)}^{*}\right\|_{\left.L_{v}^{p} \rightarrow L_{w(\cdot) k\left(\zeta^{2}(x), \cdot\right)}^{s} d x\right)^{\frac{1}{s}},}, \quad r<p\right.\end{cases}
$$

is finite. Moreover, $C_{\mathfrak{S}} \approx \mathbf{B}_{0}+\mathbf{B}_{1}+\mathbf{B}_{2}$.

\section{$6 \quad \Gamma^{p}(v) \rightarrow \Gamma^{q}(w)$ boundedness of the maximal operator}

The maximal Hardy-Littlewood operator is defined by

$$
M f(x):=\sup _{B} \frac{1}{\operatorname{mes} B} \int_{B}|f(y)| d y
$$

where the supremum is taken over all balls centered at $x \in \mathbb{R}^{n}$. The Lorentz $\Gamma$-spaces were introduced by E.T. Sawyer [32] while working on characterization of the boundedness of the maximal operator in the weighted Lorentz spaces (see also, for instance, related papers [8], [9], [14], [15], [34], [38]). More exactly, if $v \in \mathfrak{M}^{+}$and $0<p<\infty$, then

$$
\Gamma^{p}(v)=\left\{f \text { measurable on } \mathbb{R}^{n}:\left(\int_{0}^{\infty}\left[f^{* *}(x)\right]^{p} v(x) d x\right)^{\frac{1}{p}}<\infty\right\},
$$

where $f^{* *}(x):=\frac{1}{x} \int_{0}^{x} f^{*}(t) d t$ and

$$
f^{*}(t):=\inf \{s>0: \operatorname{mes}\{x:|f(x)|>s\} \leq t\} .
$$

It is known ( [1], Theorem 3.8) that

$$
[M f]^{*}(x) \approx \frac{1}{x} \int_{0}^{x} f^{*} .
$$


Therefore, $M: \Gamma^{p}(v) \rightarrow \Gamma^{q}(u)$ boundedness is equivalent to the weighted inequality

$$
\left(\int_{0}^{\infty}\left(\frac{1}{x} \int_{0}^{x}\left(\frac{1}{y} \int_{0}^{y} f\right) d y\right)^{q} u(x) d x\right)^{\frac{1}{q}} \leq C\left(\int_{0}^{\infty}\left(\frac{1}{t} \int_{0}^{t} f\right)^{p} v(t) d t\right)^{\frac{1}{p}}, f \in \mathfrak{M}^{\downarrow}
$$

restricted on the cone $\in \mathfrak{M}^{\downarrow} \subset \mathfrak{M}^{+}$of all nonincreasing functions. Moreover, the least possible constant $C$ is equivalent to the norm of $M$

$$
C \approx\|M\|_{\Gamma^{p}(v) \rightarrow \Gamma^{q}(u)}:=\sup _{0 \neq f \in \Gamma^{p}(v)} \frac{\|M f\|_{\Gamma^{q}(u)}}{\|f\|_{\Gamma^{p}(v)}} .
$$

The inequality (6.1) was first characterized in the case $1<p=q<\infty, u=v$ ( [39], Theorem 5.1) and for $1<p, q<\infty, u \neq v$ in ( [10], Theorem 3.3) and ( [33], Theorem 5.1) (see, also [11]).

Applying Theorems 3.1 and 3.2 we solve the problem for all $0<p, q<\infty$ and similar to [33] our criteria have an explicit integral form.

Let $\Omega_{1,0}:=\left\{g \in \mathfrak{M}^{\downarrow}, t g(t) \in \mathfrak{M}^{\uparrow}\right\}$. Then $F(t)=\frac{1}{t} \int_{0}^{t} f \in \Omega_{1,0}$ for any $f \in \mathfrak{M}^{\downarrow}$ and $F^{p} \in \Omega_{p, 0}:=\left\{g \in \mathfrak{M}^{\downarrow}, t^{p} g(t) \in \mathfrak{M}^{\uparrow}\right\}$. By the change $G=F^{p}$ (6.1) becomes equivalent to

$$
\left(\int_{0}^{\infty}\left(\frac{1}{x} \int_{0}^{x} G^{\frac{1}{p}}\right)^{q} u(x) d x\right)^{\frac{p}{q}} \leq C^{p} \int_{0}^{\infty} G v, G \in \Omega_{p, 0}
$$

and applying ( [33], Lemma 2.3) we reduce (6.2) to the inequality

$$
\left(\int_{0}^{\infty}\left(\frac{1}{x} \int_{0}^{x}\left(\int_{0}^{\infty} \frac{h(z) d z}{y^{p}+z^{p}}\right)^{\frac{1}{p}} d y\right)^{q} u(x) d x\right)^{\frac{p}{q}} \lesssim C^{p} \int_{0}^{\infty} h V, h \in \mathfrak{M}^{+},
$$

where

$$
V(z)=\int_{0}^{\infty} \frac{v(y) d y}{y^{p}+z^{p}}
$$

Since

$$
\int_{0}^{\infty} \frac{h(z) d z}{y^{p}+z^{p}} \approx \int_{y}^{\infty} \frac{h(z) d z}{z^{p}}+\frac{1}{y^{p}} \int_{0}^{y} h(z) d z,
$$

(6.3) is characterized by the following pair of inequalities:

$$
\left(\int_{0}^{\infty}\left(\frac{1}{x} \int_{0}^{x}\left(\int_{y}^{\infty} h(z) d z\right)^{\frac{1}{p}} d y\right)^{q} u(x) d x\right)^{\frac{p}{q}} \leq C_{1}^{p} \int_{0}^{\infty} h(t) t^{p} V(t) d t, h \in \mathfrak{M}^{+}
$$

and

$$
\left(\int_{0}^{\infty}\left(\frac{1}{x} \int_{0}^{x}\left(\int_{0}^{y} h(z) d z\right)^{\frac{1}{p}} \frac{d y}{y}\right)^{q} u(x) d x\right)^{\frac{p}{q}} \leq C_{2}^{p} \int_{0}^{\infty} h V, h \in \mathfrak{M}^{+},
$$

which are of the form (1.2) and (1.4), respectively. Moreover,

$$
C \approx C_{1}+C_{2} \text {. }
$$

Hence, applying Theorems 3.1 and 3.2, we see that

$$
C_{1} \approx \mathscr{A}_{0}+\mathscr{A}_{2}
$$


and

$$
C_{2} \approx \mathbf{A}_{0}+\mathbf{A}_{2}
$$

where the constants A's are defined by (3.1) and (3.2) for (6.4) and by (3.3) and (3.4) for (6.5) under related changes of weights, the function $\zeta$ and auxiliary operators.

Suppose for simplicity that $0<\int_{t}^{\infty} s^{-q} u(s) d s<\infty$ for all $t>0$. Now, the functions $\zeta$ and $\zeta^{-1}$ are defined by

$$
\begin{gathered}
\zeta(x):=\sup \left\{y>0: \int_{y}^{\infty} s^{-q} u(s) d s \geq \frac{1}{2} \int_{x}^{\infty} s^{-q} u(s) d s\right\}, \\
\zeta^{-1}(x):=\sup \left\{y>0: \int_{y}^{\infty} s^{-q} u(s) d s \geq 2 \int_{x}^{\infty} s^{-q} u(s) d s\right\} .
\end{gathered}
$$

For $0 \leq c<d<\infty$ and $h \in \mathfrak{M}^{+}$we put

$$
\begin{gathered}
\left(\mathscr{H}_{c, d} h\right)(x):=\chi_{(c, d]}(x) \int_{x}^{\zeta(d)} h, \\
\left(\mathscr{H}_{d} h\right)(x):=\chi_{(0, d]}(x) \int_{x}^{\infty} h, \\
\left(\mathscr{H}_{c, d}^{*} h\right)(x):=\chi_{(c, d]}(x) \int_{\zeta^{-1}(c)}^{x} h, \\
\left(\mathscr{H}_{d}^{*} h\right)(x):=\chi_{(0, d]}(x) \int_{0}^{x} h .
\end{gathered}
$$

By Theorem $3.1 \mathscr{A}_{0}$ is the least possible constant in the inequaity

$$
\left(\int_{0}^{\infty} u(x)\left(\int_{x}^{\infty} h\right)^{\frac{q}{p}} d x\right)^{\frac{p}{q}} \leq \mathscr{A}_{0}^{p} \int_{0}^{\infty} h(z) z^{p} V(z) d z, h \in \mathfrak{M}^{+}
$$

and $\mathscr{A}_{2}$ is defined by

$$
\mathscr{A}_{2}^{p}:= \begin{cases}\sup _{t \in(0, \infty)}\left(\int_{t}^{\infty} s^{-q} u(s) d s\right)^{\frac{p}{q}}\left\|\mathscr{H}_{t}\right\|_{L_{z^{p} V(z)}^{1} \rightarrow L^{\frac{1}{p}}} & p \leq q, \\ \left(\int_{0}^{\infty} x^{-q} u(x)\left(\int_{x}^{\infty} s^{-q} u(s) d s\right)^{\frac{q}{p-q}}\left\|\mathscr{H}_{\zeta^{-1}(x), \zeta(x)}\right\|_{L_{z z^{p}(z)}^{1} \rightarrow L^{\frac{1}{p}}}^{\frac{q}{p-q}} d x\right)^{\frac{p-q}{q}}, & q<p .\end{cases}
$$

Also, by Theorem $3.2 \mathbf{A}_{0}$ is the best possible constant in the inequaity

$$
\left(\int_{0}^{\infty} x^{-q} u(x)\left(\log \frac{x}{\zeta^{-2}(x)}\right)^{q}\left(\int_{0}^{\zeta^{-2}(x)} h\right)^{\frac{q}{p}} d x\right)^{\frac{p}{q}} \leq \mathbf{A}_{0}^{p} \int_{0}^{\infty} h V, h \in \mathfrak{M}^{+}
$$

and $\mathbf{A}_{2}$ is determined from

$$
\mathbf{A}_{2}^{p}:= \begin{cases}\sup _{t \in(0, \infty)}\left(\int_{t}^{\infty} s^{-q} u(s) d s\right)^{\frac{p}{q}}\left\|\mathscr{H}_{t}^{*}\right\|_{L_{V}^{1} \rightarrow L_{\frac{1}{y}}^{\frac{1}{p}},} & p \leq q, \\ \left(\int_{0}^{\infty} x^{-q} u(x)\left(\int_{x}^{\infty} s^{-q} u(s) d s\right)^{\frac{q}{p-q}}\left\|\mathscr{H}_{\zeta^{-1}(x), \zeta(x)}^{*}\right\|_{L_{V}^{1} \rightarrow L_{\frac{1}{y}}^{\frac{1}{p-q}}}^{\frac{q}{p}}\right)^{\frac{p-q}{q}}, & q<p .\end{cases}
$$


By well known results ( [18], Chapter XI, $\S ~ 1.5$, Theorem 4, see also [15], Theorem 1.1) and ( [35], Theorem 3.3) we have

$$
\mathscr{A}_{0}^{p}=\sup _{t>0} \frac{\left(\int_{0}^{t} u\right)^{\frac{p}{q}}}{t^{p} V(t)}, p \leq q
$$

and

$$
\mathscr{A}_{0}^{p} \approx\left(\int_{0}^{\infty}\left[t^{p} V(t)\right]^{\frac{q}{q-p}}\left(\int_{0}^{t} u\right)^{\frac{q}{p-q}} u(t) d t\right)^{\frac{p-q}{q}}, q<p .
$$

Analogously, we find

$$
\mathbf{A}_{0}^{p}=\sup _{t>0} \frac{\left(\int_{\zeta^{2}(t)}^{\infty} x^{-q} u(x)\left(\log \frac{x}{\zeta^{-2}(x)}\right)^{q} d x\right)^{\frac{p}{q}}}{V(t)}, p \leq q
$$

and for $q<p$

$$
\mathbf{A}_{0}^{p} \approx\left(\int_{0}^{\infty}\left(\frac{\int_{x}^{\infty} s^{-q} u(s)\left(\log \frac{s}{\zeta^{-2}(s)}\right)^{q} d s}{V\left(\zeta^{-2}(x)\right)}\right)^{\frac{q}{p-q}} x^{-q} u(x)\left(\log \frac{x}{\zeta^{-2}(x)}\right)^{q} d x\right)^{\frac{p-q}{q}}
$$

Again, applying ( [18], Chapter XI, § 1.5, Theorem 4) and ( [35], Theorem 3.3) we obtain

$$
\left\|\mathscr{H}_{t}\right\|_{L_{z^{p} V(z)}^{1} \rightarrow L^{\frac{1}{p}}}=[V(t)]^{-1}, 0<p \leq 1
$$

and

$$
\left\|\mathscr{H}_{t}\right\|_{L_{z^{p} V(z)}^{1} \rightarrow L^{\frac{1}{p}}} \approx\left(\int_{0}^{t}[V(x)]^{\frac{1}{1-p}} \frac{d x}{x}\right)^{p-1}, p>1,
$$

so that it follows from (6.6) for $p \leq q$

$$
\mathscr{A}_{2}=\sup _{t \in(0, \infty)}\left(\int_{t}^{\infty} s^{-q} u(s) d s\right)^{\frac{1}{q}}[V(t)]^{-\frac{1}{p}}, 0<p \leq 1,
$$

and

$$
\mathscr{A}_{2} \approx \sup _{t \in(0, \infty)}\left(\int_{t}^{\infty} s^{-q} u(s) d s\right)^{\frac{1}{q}}\left(\int_{0}^{t}[V(x)]^{\frac{1}{1-p}} \frac{d x}{x}\right)^{\frac{1}{p^{\prime}}}, p>1
$$

where $p^{\prime}:=\frac{p}{p-1}$. By the same way,

$$
\left\|\mathscr{H}_{\zeta^{-1}(x), \zeta(x)}\right\|_{L_{z^{p} V(z)}^{1} \rightarrow L^{\frac{1}{p}}}=\left[\frac{\zeta(x)-\zeta^{-1}(x)}{\zeta(x)}\right]^{p} \frac{1}{V(\zeta(x))}, 0<p \leq 1
$$

and

$$
\left\|\mathscr{H}_{\zeta^{-1}(x), \zeta(x)}\right\|_{L_{z^{p} V(z)}^{1} \rightarrow L^{\frac{1}{p}}} \approx\left(\int_{\zeta^{-1}(x)}^{\zeta(x)}\left[t^{p} V(t)\right]^{\frac{1}{1-p}}\left(t-\zeta^{-1}(x)\right)^{\frac{1}{p-1}} d t\right)^{p-1}, p>1
$$


Hence, from (6.6) we see that for $q<p$

$$
\mathscr{A}_{2} \approx\left(\int_{0}^{\infty} x^{-q} u(x)\left(\int_{x}^{\infty} s^{-q} u(s) d s\right)^{\frac{q}{p-q}}\left[\frac{\left.\left(\zeta(x)-\zeta^{-1}(x)\right)\right)}{\zeta(x)[V(\zeta(x))]^{\frac{1}{p}}}\right]^{\frac{p q}{p-q}} d x\right)^{\frac{p-q}{p q}}
$$

if $0<p \leq 1$ and

$$
\begin{aligned}
& \mathscr{A}_{2} \approx \\
& \left(\int_{0}^{\infty} x^{-q} u(x)\left(\int_{x}^{\infty} s^{-q} u(s) d s\right)^{\frac{q}{p-q}}\left(\int_{\zeta^{-1}(x)}^{\zeta(x)}\left(\frac{t-\zeta^{-1}(x)}{t^{p} V(t)}\right)^{\frac{1}{p-1}} d t\right)^{\frac{q(p-1)}{p-q}} d x\right)^{\frac{p-q}{p q}},
\end{aligned}
$$

when $p>1$.

Similarly,

$$
\left\|\mathscr{H}_{t}^{*}\right\|_{L_{V}^{1} \rightarrow L_{\frac{1}{y}}^{\frac{1}{p}}}=\sup _{s \in(0, t)}[V(s)]^{-1}\left(\log \frac{t}{s}\right)^{p}, 0<p \leq 1
$$

and

$$
\left\|\mathscr{H}_{t}^{*}\right\|_{L_{V}^{1} \rightarrow L_{\frac{1}{y}}^{\frac{1}{p}}} \approx\left(\int_{0}^{t}[V(x)]^{\frac{1}{1-p}}\left(\log \frac{t}{s}\right)^{\frac{1}{p-1}} \frac{d x}{x}\right)^{p-1}, p>1 .
$$

Now, it follows from (6.7) for $p \leq q$

$$
\mathbf{A}_{2}=\sup _{t \in(0, \infty)}\left(\int_{t}^{\infty} s^{-q} u(s) d s\right)^{\frac{1}{q}} \sup _{s \in(0, t)}[V(s)]^{-\frac{1}{p}} \log \frac{t}{s}, 0<p \leq 1
$$

and

$$
\mathbf{A}_{2} \approx \sup _{t \in(0, \infty)}\left(\int_{t}^{\infty} s^{-q} u(s) d s\right)^{\frac{1}{q}}\left(\int_{0}^{t}[V(x)]^{\frac{1}{1-p}}\left(\log \frac{t}{s}\right)^{\frac{1}{p-1}} \frac{d x}{x}\right)^{\frac{1}{p^{\prime}}}, p>1,
$$

We have

$$
\left\|\mathscr{H}_{\zeta^{-1}(x), \zeta(x)}^{*}\right\|_{L_{V}^{1} \rightarrow L_{\frac{1}{y}}^{\frac{1}{p}}}=\sup _{s \in\left(\zeta^{-1}(x), \zeta(x)\right)} \frac{\left(\log \frac{\zeta(x)}{s}\right)^{p}}{V(s)}, 0<p \leq 1
$$

and

$$
\left\|\mathscr{H}_{\zeta^{-1}(x), \zeta(x)}^{*}\right\|_{L_{V}^{1} \rightarrow L_{\frac{1}{y}}^{\frac{1}{p}}} \approx\left(\int_{\zeta^{-1}(x)}^{\zeta(x)}[V(t)]^{\frac{1}{1-p}}\left(\log \frac{\zeta(x)}{t}\right)^{\frac{1}{p-1}} \frac{d t}{t}\right)^{p-1}, p>1 .
$$

Thus, from (6.7) we find for $q<p$

$$
\mathbf{A}_{2} \approx\left(\int_{0}^{\infty} x^{-q} u(x)\left(\int_{x}^{\infty} s^{-q} u(s) d s\right)^{\frac{q}{p-q}}\left[\sup _{s \in\left(\zeta^{-1}(x), \zeta(x)\right)} \frac{\left(\log \frac{\zeta(x)}{s}\right)^{p}}{V(s)}\right]^{\frac{q}{p-q}} d x\right)^{\frac{p-q}{p q}}
$$


if $0<p \leq 1$ and

$$
\begin{aligned}
& \mathbf{A}_{2} \approx \\
& \left(\int_{0}^{\infty} x^{-q} u(x)\left(\int_{x}^{\infty} s^{-q} u(s) d s\right)^{\frac{q}{p-q}}\left(\int_{\zeta^{-1}(x)}^{\zeta(x)}\left(\frac{\log \frac{\zeta(x)}{t}}{V(t)}\right)^{\frac{1}{p-1}} \frac{d t}{t}\right)^{\frac{q(p-1)}{p-q}} d x\right)^{\frac{p-q}{p q}}
\end{aligned}
$$

when $p>1$.

Finally, we obtain the following.

Theorem 6.1. Let $0<p, q<\infty$. Then for the maximal Hardy-Littlewood operator

$$
\|M\|_{\Gamma^{p}(v) \rightarrow \Gamma^{q}(u)} \approx \mathscr{A}_{0}+\mathscr{A}_{2}+\mathbf{A}_{0}+\mathbf{A}_{2},
$$

where the constants on the right-hand side are determined by (6.8)-(6.11) for $\mathscr{A}_{0}$ and $\mathbf{A}_{0}$ and by (6.12)-(6.19) for $\mathscr{A}_{2}$ and $\mathbf{A}_{2}$.

\section{References}

[1] C. Bennett and R. Sharpley, "Interpolation of operators", Pure and Applied Mathematics 129, Academic Press, Inc., Boston, MA, 1988.

[2] S. Bloom and R. Kerman, Weighted norm inequalities for operators of Hardy type, Proc. Amer. Math. Soc. 113 (1991), 135-141.

[3] V.I. Burenkov and H.V. Guliev, Necessary and sufficient conditions for boundedness of the maximal operator in the local Morry-type spaces, Studia Math. 163 (2004), 157-176.

[4] V.I. Burenkov, A. Gogatishvili, V.S. Guliev and R.Ch. Mustafayev, Necessary and sufficient conditions for boundedness of the fractional maximal operator in the local Morry-type spaces, J. Comput. Appl. Math. 208 (2007), 280-301.

[5] V.I. Burenkov, A. Gogatishvili, V.S. Guliev and R.Ch. Mustafayev, Necessary and sufficient conditions for boundedness of the Riesz potential in the local Morry-type spaces, Potential Anal. 30 (2009), 211-249.

[6] V.I. Burenkov, P. Jain and T.V. Tararykova, On boundedness of the Hardy operator in Morry-type spaces, Eurasian Math. J. 2 (2011), 52-80.

[7] V.I. Burenkov and R. Oinarov, Necessary and sufficient conditions for boundedness of the Hardy-type operator from a weighted Lebesgue space to a Morry-type spaces, Math. Inequal. Appl. 16 (2013), 1-19.

[8] M. Carro, L. Pick, J. Soria and V.D. Stepanov, On embeddings between classical Lorentz spaces, Math. Inequal. Appl. 4 (2001), 397-428.

[9] M. Carro and J. Soria, Boundedness of some integral operators, Canad. J. Math. 45 (1993), 1155-1166.

[10] M.L. Goldman, H.P. Heinig and V.D. Stepanov, On the principle of duality in Lorentz spaces, Canad. J. Math. 48 (1996), 959-979. 
[11] M.L. Goldman and M.V. Sorokina, Three-weighted Hardy-type inequalities on the cone of quasimonotone functions, Doklady Math. 71 (2005), 209-213.

[12] A. Gogatishvili, B. Opic and L. Pick, Weighted inequalities for Hardy-type operators involving suprema, Collect. Math. 57 (2006), 227-255.

[13] A. Gogatishvili and L. Pick, A reduction theorem for supremum operators, J. Comp. Appl. Math. 208 (2007), 270-279.

[14] A. Gogatishvili and V.D. Stepanov, Reduction theorems for operators on the cones of monotone functions, J. Math. Anal. Appl. 405 (2013), 156-172.

[15] A. Gogatishvili and V.D. Stepanov, Reduction theorems for weighted integral inequalities on the cone of monotone functions, Russian Math. Surveys, 68(4) (2013), 597-664.

[16] A. Gogatishvili, R. Mustafayev and L.-E. Persson, Some new iterated Hardy-type inequalities, J. Function Spaces Appl. 2013, Art. ID 734194, 30 pp.

[17] A. Gogatishvili, R. Mustafayev and L.-E. Persson, Some new iterated Hardy-type inequalities: the case $\theta=1$, J. Inequal. Appl. 2013, 2013:515.

[18] L.V. Kantorovich and G.P. Akilov, Functional Analysis, Pergamon Press, Oxford, 1982.

[19] Q. Lai, Weighted modular inequalities for Hardy-type operators, Proc. London Math. Soc. 79 (1999), 649-672.

[20] E.N. Lomakina and V.D. Stepanov, On the Hardy-type integral operators in Banach function spaces, Publ. Mat. 42 (1998), 165-194.

[21] R. Oinarov, Two-sided estimates of the norm of some classes of integral operators, Proc. Steklov Inst. Math. 204 (1994), 205-214.

[22] D.V. Prokhorov, Weighted Hardy's inequalities for negative indices, Publ. Mat. 48 (2004), 423-443.

[23] D.V. Prokhorov, Inequalities of Hardy type for a class of integral operators with measures, Anal. Math. 33 (2007), 199-225.

[24] D.V. Prokhorov, Inequalities for Riemann-Liouville operator involving suprema, Collect. Math. 61 (2010), 263-276.

[25] D.V. Prokhorov, Lorentz norm inequalities for the Hardy operator involving suprema, Proc. Amer. Math. Soc. 140 (2012), 1585-1592.

[26] D.V. Prokhorov, On a weighted Hardy-type inequality, Doklady Math. 88 (2013), 687689.

[27] D.V. Prokhorov, Boundedness and compactness of a supremum-involving integral operator, Proc. Steklov Inst. Math. 283 (2013), 136-148.

[28] D.V. Prokhorov and V.D. Stepanov, Weighted estimates of Riemann-Liouville operators and applications, Proc. Steklov Inst. Math. 243 (2003), 278-301.

[29] D.V. Prokhorov and V.D. Stepanov, On supremum operators, Doklady Math., 84 (2011), 457-458. 
[30] D.V. Prokhorov and V.D. Stepanov, Weighted estimates for a class of sublinear operators, Doklady Math., 88 (2013), 721-723.

[31] D.V. Prokhorov and V.D. Stepanov, On weighted Hardy inequalities in mixed norms, Proc. Steklov Inst. Math. 283 (2013), 149-164.

[32] E. Sawyer, Boundedness of classical operators on classical Lorentz spaces, Studia Math., 96 (1990), 145-158.

[33] G. Sinnamon, Embeddings of concave functions and duals of Lorentz spaces, Publ. Mat. 46 (2002), 489-515.

[34] G. Sinnamon, Transferring monotonicity in weighted norm inequalities, Collect. Math. 54 (2003), 181-216.

[35] G. Sinnamon and V.D. Stepanov, The weighted Hardy inequality: new proofs and the case $\mathrm{p}=1$. J. London Math. Soc. 54 (1996), 89-101.

[36] V.D. Stepanov, Weighted norm inequalities of Hardy type for a class of integral operators, J. London Math. Soc. 50 (1994), 105-120.

[37] V.D. Stepanov, On a supremum operator, Spectral Theory, Function Spaces and Inequalities. Operator Theory: Advances and Applications. 219 Birkhäuser. Basel. 2012, 233-242.

[38] V.D. Stepanov, The weighted Hardy's inequality for nonincreasing functions. Trans. Amer. Math. Soc. 338 (1993), 173-186.

[39] V.D. Stepanov, Integral operators on the cone of monotone functions. J. London Math. Soc. 48 (1993), 465-487.

[40] V.D. Stepanov and E.P. Ushakova, Alternative criteria for the boundedness of Volterra integral operators in Lebesgue spaces, Math. Inequal. Appl. 12 (2009), 873-889.

[41] V.D. Stepanov and E.P. Ushakova, Kernel operators with variable intervals of integration in Lebesgue spaces and applications, Math. Inequal. Appl. 13 (2010), 449-510.

Dmitrii V. Prokhorov, Computing Centre FEB RAS, Khabarovsk, Russia. E-mail address: prohorov@as.khb.ru

Vladimir D. Stepanov, Steklov Institute of Mathematics; Peoples Friendship University of Russia, Moscow, Russia. E-mail address: stepanov@mi.ras.ru 